\title{
Platelet membrane-camouflaged silver metal-organic framework drug system against infections caused by methicillin-resistant Staphylococcus aureus
}

\author{
Rong Huang ${ }^{1,2}$, Guang-Qing Cai ${ }^{3}$, Jian Li ${ }^{1}$, Xi-Sheng Li ${ }^{2}$,Hai-Ting Liu' ${ }^{1}$, Xue-Ling Shang ${ }^{2}$, Jian-Dang Zhou²,
} Xin-Min Nie $2^{2^{*}}$ and Rong Gui ${ }^{*}$

\begin{abstract}
Background: Due to the intelligent survival strategy and self-preservation of methicillin-resistant Staphylococcus aureus (MRSA), many antibiotics are ineffective in treating MRSA infections. Nano-drug delivery systems have emerged as a new method to overcome this barrier. The aim of this study was to construct a novel nano-drug delivery system for the treatment of MRSA infection, and to evaluate the therapeutic effect and biotoxicity of this system. We prepared a nano silver metal-organic framework using 2-methylimidazole as ligand and silver nitrate as ion provider. Vancomycin (Vanc) was loaded with Ag-MOF, and nano-sized platelet vesicles were prepared to encapsulate Ag-MOFVanc, thus forming the novel platelet membrane-camouflaged nanoparticles PLT@Ag-MOF-Vanc.

Results: The synthesized Ag-MOF particles had uniform size and shape of radiating corona. The mean nanoparticle size and zeta potential of PLT@Ag-MOF-Vanc were $148 \mathrm{~nm}$ and $-25.6 \mathrm{mV}$, respectively. The encapsulation efficiency (EE) and loading efficiency (LE) of vancomycin were 81.0 and 64.7\%, respectively. PLT@Ag-MOF-Vanc was shown to be a pH-responsive nano-drug delivery system with good biocompatibility. Ag-MOF had a good inhibitory effect on the growth of three common clinical strains (Escherichia coli, Pseudomonas aeruginosa, and S. aureus). PLT@Ag-MOF-Vanc showed better antibacterial activity against common clinical strains in vitro than free vancomycin. PLT@Ag-MOF-Vanc killed MRSA through multiple approaches, including interfering with the metabolism of bacteria, catalyzing reactive oxygen species production, destroying the integrity of cell membrane, and inhibiting biofilm formation. Due to the encapsulation of the platelet membrane, PLT@Ag-MOF-Vanc can bind to the surface of the MRSA bacteria and the sites of MRSA infection. PLT@Ag-MOF-Vanc had a good anti-infective effect in mouse MRSA pneumonia model, which was significantly superior to free vancomycin, and has no obvious toxicity.
\end{abstract}

Conclusions: PLT@Ag-MOF-Vanc is a novel effective targeted drug delivery system, which is expected to be used safely in anti-infective therapy of MRSA.

Keywords: Platelet, Metal-organic framework, Vancomycin, Methicillin-resistant, Staphylococcus aureus

*Correspondence: niexinmin@csu.edu.cn; guirong@csu.edu.cn

1 Department of Blood Transfusion, The Third Xiangya Hospital, Central South University, Hunan, Changsha, China

2 Department of Laboratory Medicine, The Third Xiangya Hospital, Central South University, Hunan, Changsha, China

Full list of author information is available at the end of the article

\section{Introduction}

Staphylococcus aureus (S. aureus) is a kind of gram-positive bacteria with strong pathogenicity. It can cause infections of the skin and soft tissues, and internal organs; it is also the most common gram-positive bacterial species 
that causes sepsis [1]. More severe cases may progress to multiple organ failure, diffuse intravascular coagulation, lactic acidosis, and even death. Antibiotics are the first-line drugs in bacterial infection treatment; however, due to the abuse of antibiotics and long-term natural selection of bacteria, drug-resistant bacteria appeared, posing a major threat to the existing antibiotics in clinical practice [2]. At present, more than $90 \%$ of S. aureus clinical isolates are resistant to penicillin [3]. To overcome penicillin resistance, scientists developed methicillin, but within two years of using methicillin, in 1961, Jevons found methicillin-resistant Staphylococcus aureus (MRSA) for the first time in the UK [4]. MRSA strains were resistant not only to methicillin, but to all penicillins, cephalosporins, and carbapenems [5], making MRSA more harmful than those bacteria that were resistant to "only" one kind of antibiotic. In addition, MRSA strains have become resistant to more and more other antibiotics, such as fluoroquinolones, macrolides, aminoglycosides, and clindamycin [6-9]. According to the US Centers for Disease Control and Prevention (CDC), MRSA has been considered as a serious threat after taking into account various factors, such as treatability, mortality, burden on the healthcare-infrastructure and the community, prevalence and increasing trends of resistance [10]. MRSA remains one of the difficult-to-treat ESKAPE pathogens (Enterococcus faecium, S. aureus, Klebsiella pneumoniae, Acinetobacter baumannii, Pseudomonas aeruginosa, and Enterobacter species), which is the most serious threat to the development of untreatable multidrug-resistant (MDR) infections [11, 12].

MRSA has developed multiple resistance mechanisms, including the thickened bacterial cell wall, the increased efflux pumps on cell membrane, the drug targets mutations, drugs modifications and the altered bacterial colonization state $[3,13-15]$. Thus, clinical treatment of MRSA infection is a challenging problem. The main drawbacks of current antibiotics are associated side effects and increasing resistance. Although vancomycin remains the first-line therapy of choice, it has some limitations, such as high doses, slow antimicrobial rate, nephrotoxicity, low tissue penetration, and low oral absorption [16-18]. Rising resistance has also shaken faith in vancomycin $[19,20]$. In addition to vancomycin, daptomycin is an alternative treatment which can circumvent resistance that occurs in MRSA treatment [21, 22]. Daptomycin may be used for MRSA bacteremia, but not for pneumonia of lower respiratory, and is associated with eosinophilic pneumonia and rhabdomyolysis [23, 24]. Furthermore, emergence of resistance to daptomycin during therapy was a well-described phenomenon that threatens the clinical use of this antibiotic, further limiting the therapeutic options against MRSA $[25,26]$.
Combination antibiotic therapy is an under-explored new hope but not always effective [22, 27]. Moreover, MRSA antibiotic treatments have also been shown to be non-specific, as they kill beneficial human bacteria, leading to dysbiosis (microbial imbalance) [28].

Current antibiotics against MRSA have either failed or shown severe side effects, making it necessary to find alternative strategies in addition to the discovery of novel drug and the reuse of current drugs. Using nanomaterial approach to combat the antibiotic resistance could offer new opportunities to address these challenges [29]. Nanomaterials have unique advantages including small particle size, high surface area, high drug carrying capacity and targeting ability, which can be used for advanced delivery of antibiotics or other antibacterial drugs. Nanomaterials could effectively improve the permeability of the cell membrane for loaded drugs, enhance intracellular drug accumulation, improve the antibacterial activity of antibiotics against drug-resistant strains, provide a variety of bactericidal mechanisms, and inhibit the formation of S. aureus biofilm [30].

Some metal-based nanoparticles have natural antimicrobial activity and therefore show great potential for antimicrobial therapy [31]. Silver is one of the most effective and commonly used antibacterial materials, which can interfere with key functions in antimicrobial resistant microorganisms [32, 33]. Silver breaks down the cell wall by interacting with the proteins of cell wall, inhibits cell division by interacting with DNA and RNA, interferes with signal transduction, and leads to the production of ROS $[34,35]$. In view of antibiotics overuse leading to the increase of drug-resistant strains, silver nanoparticles (AgNPs) have emerged as a potential antibacterial agent. Combined use of AgNPs with antibiotics is a known strategy to overcome multiple drug resistance, which can enhance the overall antibacterial activiy [18, 36, 37]. It also allows to reduce the dosages of the antibiotics and attenuate side effects [38, 39]. Metal-organic frameworks (MOFs) are polymers assembled by metal ions and organic ligands through coordination. Coordination polymers contain many metal ions and ligands and they have flexible structure and unique properties; moreover, they are organic-inorganic hybrid materials with high porosity and specific surface area [40]. Therefore, in this study, we used 2-methylimidazole as a ligand and silver nitrate as an ion provider to prepare a nano metalorganic framework named Ag-MOF, which was loaded with vancomycin to treat MRSA infection through a dual antibacterial mechanism.

In view of the short half-life of exogenous nanoparticles in the circulatory system, natural biofilm was used to camouflage nanoparticles in this study. Among natural biological carriers, platelets (PLT) - autologous blood 
cells-have good biocompatibility, with which synthetic carriers cannot compete [41]. Not only can platelet membrane-camouflaged nanoparticles significantly reduce the macrophage uptake and particle-induced complement activation, but also they still show platelet-like function after camouflaging, which can effectively solve the plasma protein absorption on nanomaterial surface [42, 43]. In addition, platelets are the first and most abundant cell type accumulated in the intravascular infection and have the ability to recognize inflammatory cells $[44,45]$. The inflammatory endothelial cells express P-selectin and E-selectin; the platelet membrane can express VWF, ICAM-1, and P-selectin receptor GPIB $\alpha$, thus enabling platelets to bind with the inflammatory endothelial cells [46]. S. aureus can bind to platelets through adhesins such as protein A, agglutinin A, agglutinin B, fibronectin A, and serine-rich surface proteins $[47,48]$. Therefore, platelet membrane-camouflaged nanoparticles is expected to enhance the efficacy of existing drugs by protecting them from degradation; moreover, it can also increase the local drug concentration in infection site by providing targeted drug delivery and reinforce bactericidal effect. Nanoparticles targeting the infection site can deliver high concentration of antimicrobial drugs at the site of infection, while maintain a low total dose of drug administered, thus reducing the dose of antibiotics used and reducing toxicity to non-targeted organs.

In this study, nano-sized platelet vesicles were prepared to encapsulate Ag-MOF-Vanc, thereby forming the novel platelet membrane-camouflaged nanoparticles PLT@AgMOF-Vanc (Fig. 1). The PLT@Ag-MOF-Vanc synthesized in our study had a high drug loading rate, good biocompatibility, dual antibacterial mechanism, and targeting; it also has the ability to effectively improve the antibacterial activity of vancomycin, with no obvious toxicity.

\section{Results and discussion}

Preparation and characterization of PLT@Ag-MOF-Vanc

As shown in Fig. 1, the preparation of PLT@Ag-MOFVanc mainly included the following three steps: (1) synthesis of $\mathrm{Ag}-\mathrm{MOF}$ with 2-methylimidazole and $\mathrm{AgNO}_{3}$ in a high temperature reactor; (2) loading vancomycin to Ag-MOF to synthesize Ag-MOF-Vanc; (3) adding PLT membrane (PLTm) vesicles to encapsulate Ag-MOF-Vanc

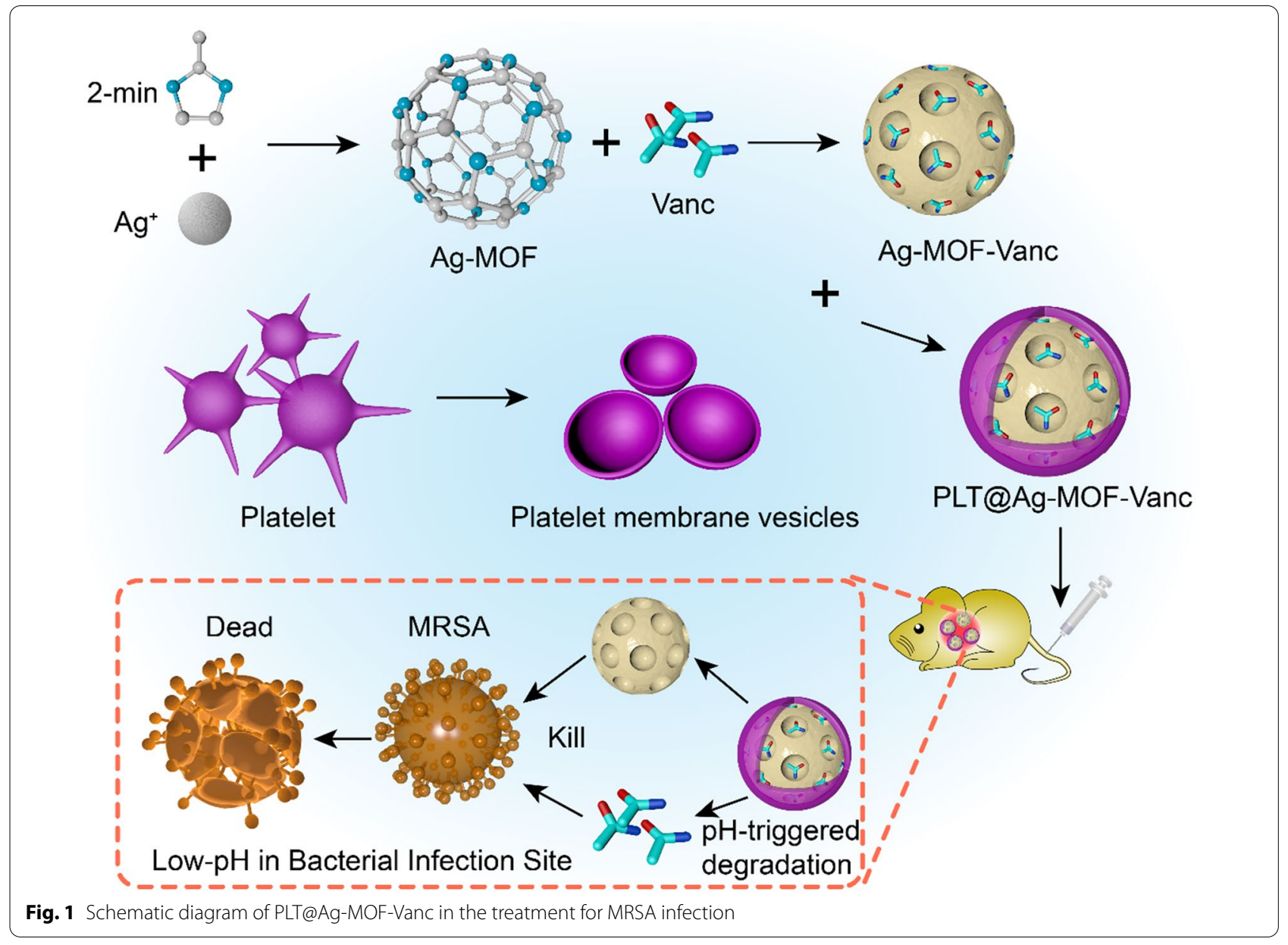


to form the final product PLT@Ag-MOF-Vanc. Transmission electron microscopy (TEM) (Fig. 2a) showed that the synthesized Ag-MOF particles had uniform size $(130-150 \mathrm{~nm})$ and shape of radiating corona. After the fusion with PLTm vesicles, Ag-MOF was observed to be encapsulated into PLTm vesicles. EDS element mapping for PLT@Ag-MOF (Fig. 2b) showed the colocalization of Ag atoms with elements (O and S) in PLTm, which could confirm the encapsulation of Ag-MOF by PLTm. The protein composition of PLTm vesicles and PLT@AgMOF-Vanc was detected by sodium dodecyl sulfate polyacrylamide gel electrophoresis(SDS-PAGE). The results showed that PLT@Ag-MOF-Vanc and PLTm vesicles had similar protein profiles, which also confirmed the successful transfer of PLTm proteins to PLT@Ag-MOF-Vanc (Fig. 2c). Dynamic light scattering (DLS) data showed that the particle size of Ag-MOF was about $133 \mathrm{~nm}$. After the encapsulation by PLTm vesicles, the particle size of PLT@Ag-MOF-Vanc was about 148 nm and was close to the size of PLTm vesicles (146 nm, Fig. 2d), which was comparable with the TEM results. Zeta potential of AgMOF, PLTm, and PLT@Ag-MOF-Vanc were - $19.0 \mathrm{mV}$, $-18.6 \mathrm{mV}$, and $-25.6 \mathrm{mV}$, respectively (Fig. $2 \mathrm{e}$ ).

UV-vis spectrum analysis showed that PLT@Ag-MOFVanc had three characteristic absorption peaks, located at $199 \mathrm{~nm}, 213 \mathrm{~nm}$, and $281 \mathrm{~nm}$, which were consistent with the characteristic absorption peaks of PLTm vesicles, Ag-MOF, and vancomycin, respectively (Fig. 2f). In the FTIR spectra of Ag-MOF (Fig. 2 g), the absorption peaks of imidazole ring in 2-methylimidazole all shifted to different degrees. $\mathrm{C}-\mathrm{H}$ stretching vibration in the imidazole ring was shifted from $3137 \mathrm{~cm}^{-1}$ to $3122 \mathrm{~cm}^{-1}$, $\mathrm{C}-\mathrm{N}$ stretching vibration in the imidazole ring was shifted from $1597 \mathrm{~cm}^{-1}$ to $1612 \mathrm{~cm}^{-1}$, and the stretching vibration of the imidazole ring was shifted from $1448 \mathrm{~cm}^{-1}$ to $1467 \mathrm{~cm}^{-1}$.This indicated that $\mathrm{Ag}$ ion in $\mathrm{AgNO}_{3}$ reacted with $\mathrm{N}$ in dimethyl imidazole to form Ag-N chemical bond, which changed the infrared spectrum absorption peak of imidazole ring in 2-methylimidazole. The $\mathrm{N}-\mathrm{H}$ stretching vibration peak in the original 2-methylimidazole disappeared at $3179 \mathrm{~cm}^{-1}$, indicating that Ag may have reacted with the $\mathrm{N}-\mathrm{H}$ in the 2-methylimidazole.

\section{Drug loading and release of PLT@Ag-MOF-Vanc}

MOF is an ideal drug carrier due to its high porosity and specific surface area. In this study, vancomycin was loaded in Ag-MOF, and the encapsulation efficiency
(EE) and loading efficiency (LE) of vancomycin were 81.0 and $64.7 \%$, respectively (Fig. 3a). The ideal drug carrier should efficiently load the drug but also reach specific sites to achieve responsive release. The $\mathrm{pH}$ values of the infected site and intracellular environment were lower than those of healthy tissue and extracellular environment, respectively [30, 49]. Therefore, $\mathrm{pH}$-sensitive nanoparticles could have a better inhibitory effect on the infection of S. aureus. In order to verify the $\mathrm{pH}$-responsive release of PLT@Ag-MOF-Vanc in infected microenvironment, $\mathrm{pH} 7.4$ and $\mathrm{pH} 6.5$ were used in this study to simulate neutral blood circulation environment and the acidic infection microenvironment, respectively. As shown in Fig. 3b, vancomycin was more easily released at $\mathrm{pH} 6.5$ than at $\mathrm{pH}$ 7.4. It is beneficial for vancomycin in PLT@Ag-MOF-Vanc to be released at the infected site rather than in neutral circulation, indicating that PLT@ Ag-MOF-Vanc can be used for drug delivery, especially at the infected site. Similar to vancomycin, the release rate of $\mathrm{Ag}^{+}$in $\mathrm{Ag}-\mathrm{MOF}$ increased with the decrease in $\mathrm{pH}$ value (Fig. 3c). In summary, $\mathrm{Ag}^{+}$and vancomycin in PLT@Ag-MOF-Vanc can be released rapidly in the weak acidic environment of the infected area. In addition, the cumulative release rate of $\mathrm{Ag}^{+}$and vancomycin in PLT@ Ag-MOF-Vanc was lower than that in Ag-MOF-Vanc, indicating that PLTm inhibited the rapid release of the drug to some extent and played a role in continuous release. These results indicate that PLT@Ag-MOF-Vanc is an effective drug carrier.

\section{Biocompatibility of PLT@Ag-MOF-Vanc}

To evaluate whether PLT@Ag-MOF-Vanc was endowed with good blood compatibility, hemolysis test was performed. Namely, $5 \%$ erythrocytes were incubated with different concentrations of Ag-MOF and PLT@Ag-MOF $(0,5,10,20,40,80$, and $160 \mu \mathrm{g} / \mathrm{mL})$ for $2 \mathrm{~h}$. As shown in Fig. 4a, Ag-MOF and PLT@Ag-MOF did not cause significant hemolysis during $2 \mathrm{~h}$ (less than $3 \%$ for both). The results indicated that Ag-MOF itself had good blood compatibility, and could be maintained after PLTm encapsulation.

To demonstrate the immune escape ability of PLTmcamouflaged PLT@Ag-MOF-Vanc, the phagocytosis of RAW264.7 macrophages was evaluated by confocal laser scanning microscope (CLSM). The green fluorescence of Ag-MOF was used for cell imaging. As shown in Figs. 4b and $5 \mathrm{~h}$ after injection of Ag-MOF-Vanc, a large number

(See figure on next page.)

Fig. 2 Characterization of PLT@Ag-MOF-Vanc. a TEM images of Ag-MOF, PLTm, and PLT@Ag-MOF. b TEM image and corresponding element mapping of PLT@Ag-MOF. Scale bar: 100 nm. c SDS-PAGE protein assessment. d Particle sizes and e zeta potential values of Ag-MOF, PLTm, and PLT@Ag-MOF.f UV-vis spectra of Vanc, Ag-MOF, PLTm, and PLT@Ag-MOF-Vanc. g FTIR spectra of Ag-MOF, 2-methylimidazole and AgNO 3 . Data are presented as means $\pm S D(n=3)$ 


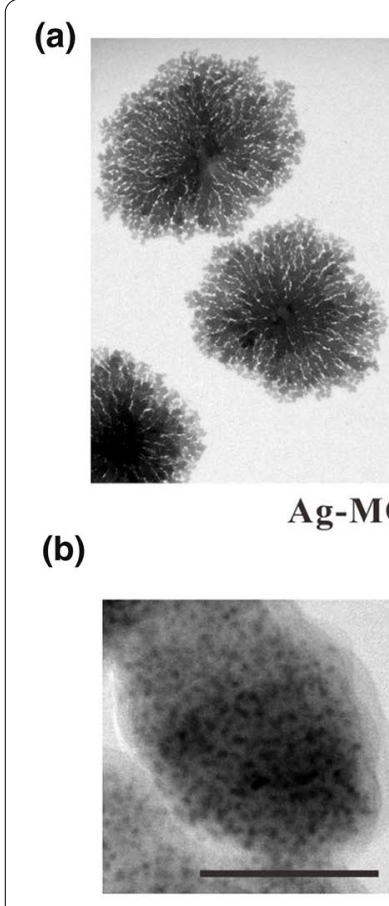

(c)

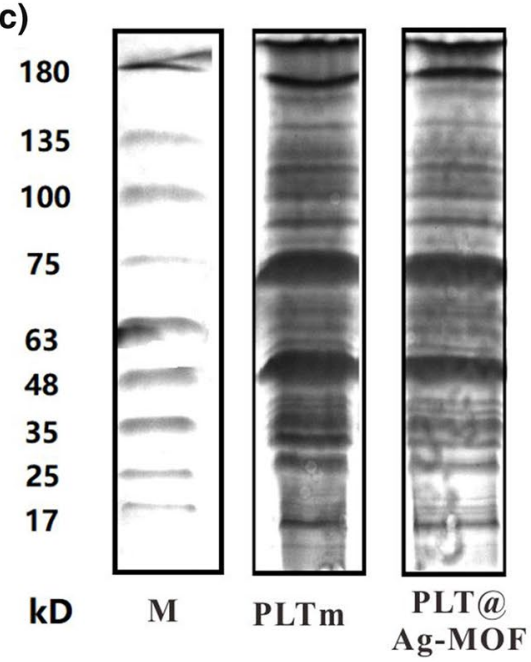

(f)

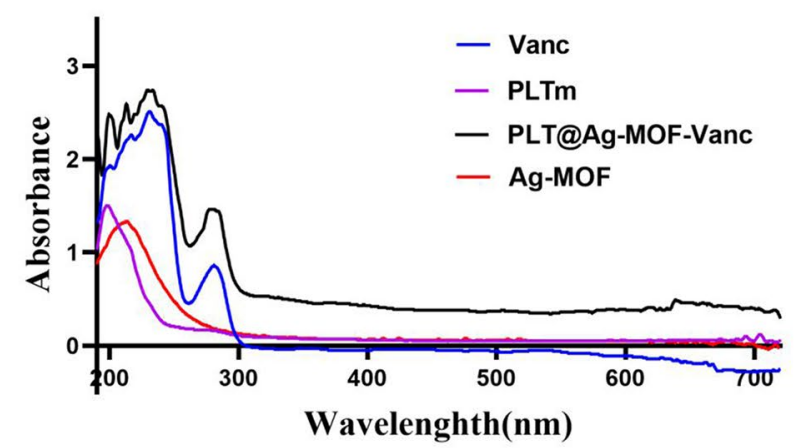

Fig. 2 (See legend on previous page.)

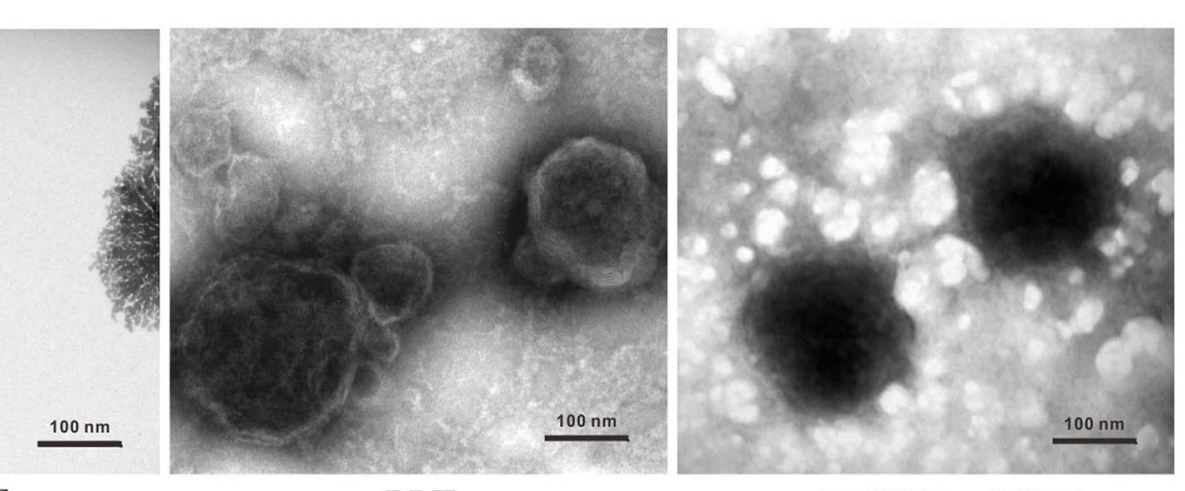

PLTm

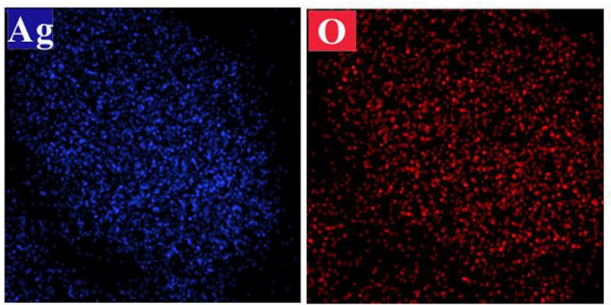

(d)

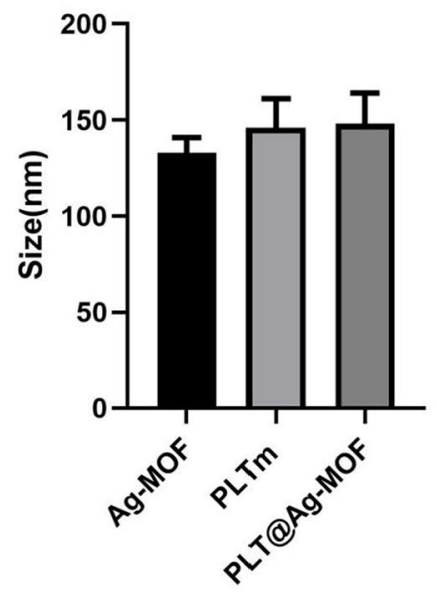

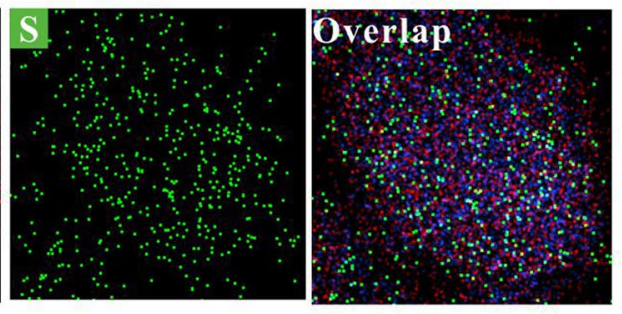

(e)

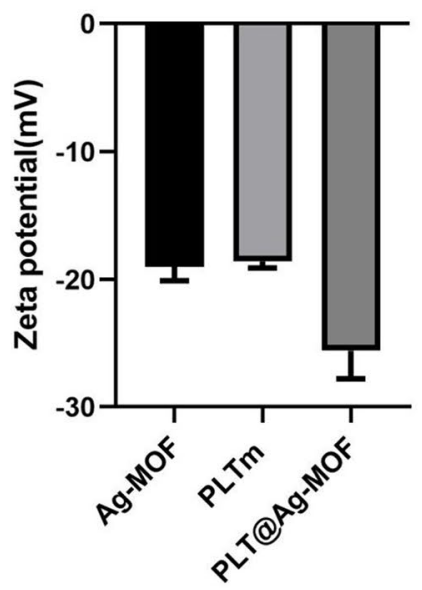

(g)

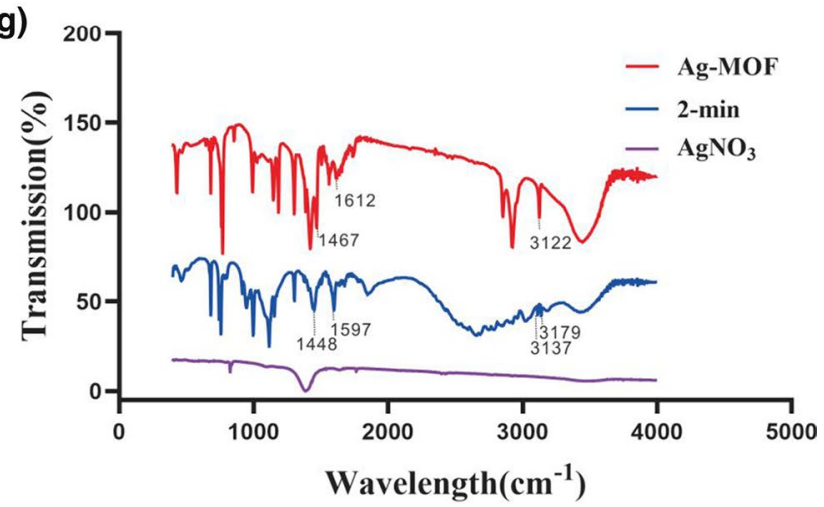



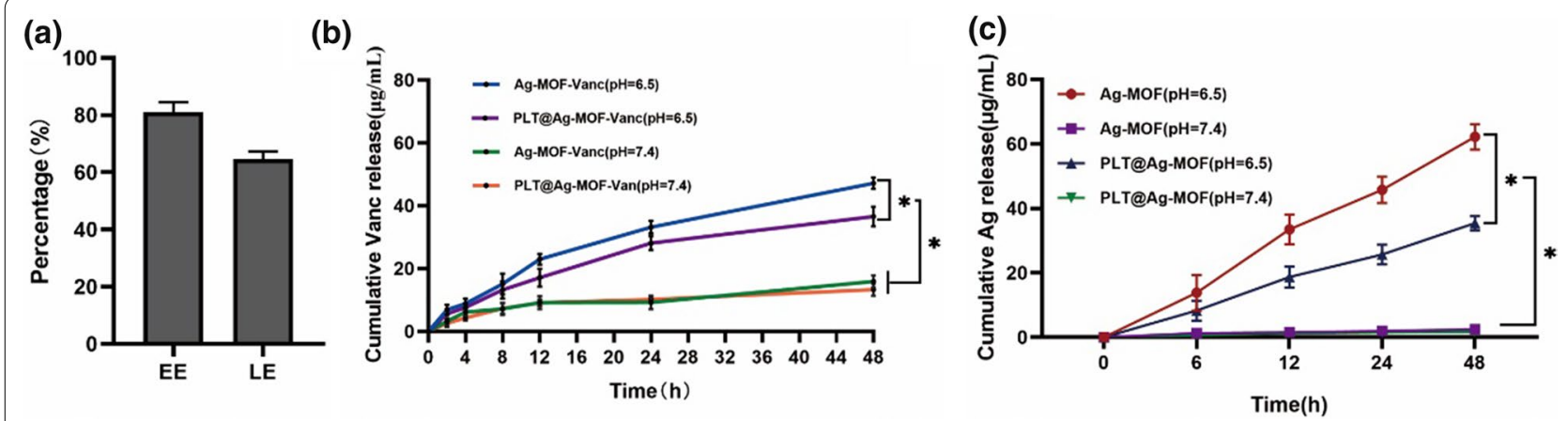

Fig. 3 Drug loading and release of PLT@Ag-MOF-Vanc. a EE and LE of PLT@Ag-MOF-Vanc. b Cumulative release rates of vancomycin from Ag-MOF-Vanc or PLT@Ag-MOF-Vanc at different pH values (6.5 and 7.4).c Cumulative release rates of Ag from Ag-MOF-Vanc or PLT@Ag-MOF-Vanc at different $\mathrm{pH}$ values (6.5 and 7.4). Data are presented as means $\pm \mathrm{SD}(\mathrm{n}=3) .{ }^{*} \mathrm{p}<0.05$

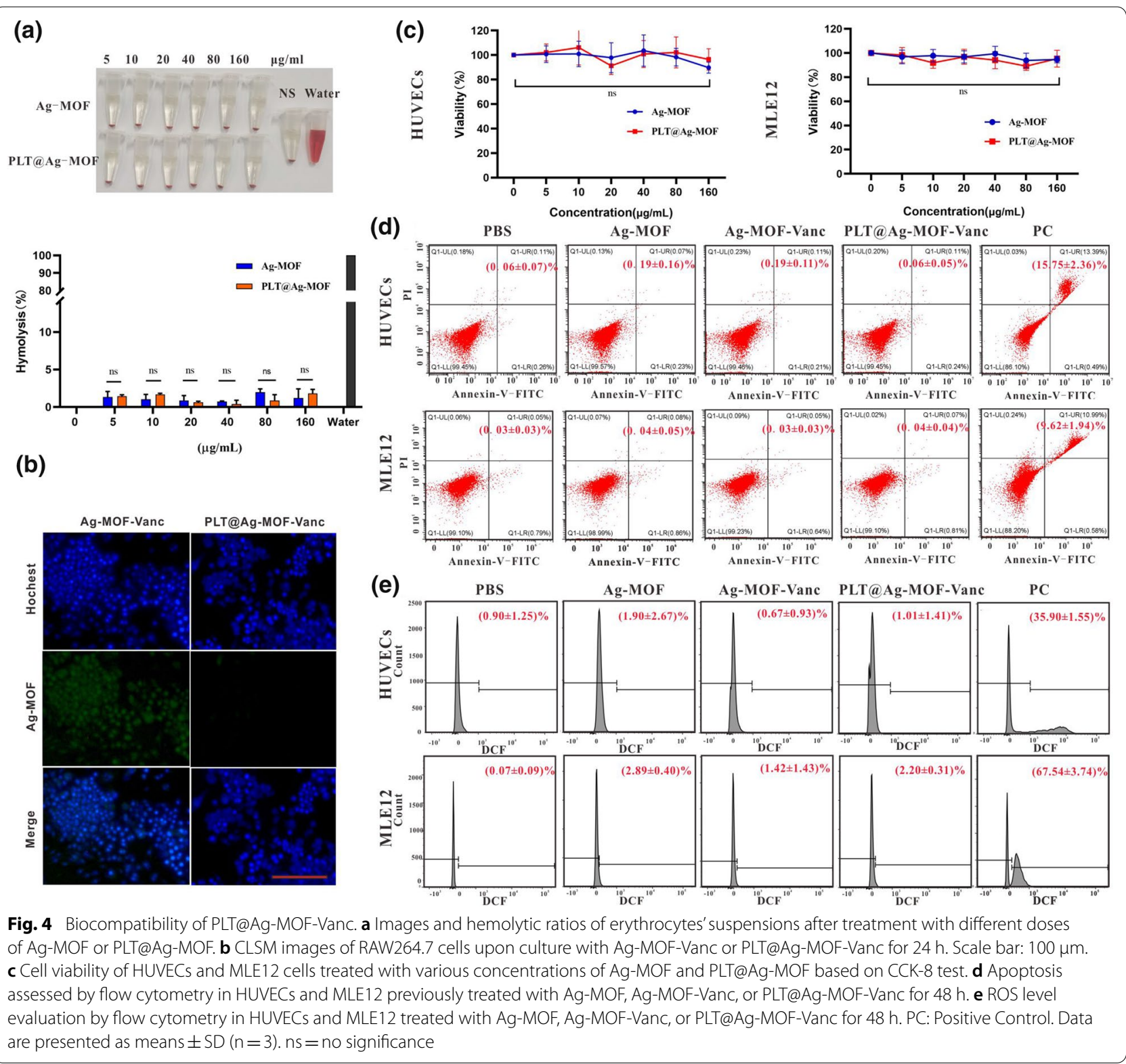


of Ag-MOF was engulfed by RAW264.7 cells. Meanwhile, under the same conditions, the green fluorescence in RAW264.7 cells treated with PLT@Ag-MOF-Vanc was significantly reduced; these data indicated that after being encapsulated by PLTm vesicles, the immunogenicity of PLT@Ag-MOF-Vanc decreased and was not recognized as a non-self-component by macrophages, so the phagocytosis was effectively inhibited. These above characteristics endowed PLT@Ag-MOF-Vanc with prolonged circulatory half-life by reducing recognition and clearance by phagocytes from the reticuloendothelial system in vivo.

To further evaluate the cytotoxicity of the material, HUVECs and MLE12 cells were treated with different concentrations $(0,5,10,20,40,80$, and $160 \mu \mathrm{g} / \mathrm{mL})$ of Ag-MOF and PLT@Ag-MOF, and the cell vitality was detected by CCK-8. As shown in Fig. 4c, after 48 h of mixed culture with the added material, cell vitality did not significantly decrease; therefore, Ag-MOF and PLT@ Ag-MOF have no obvious cytotoxicity. In this study, the effects of the materials on cell apoptosis and reactive oxygen species (ROS) production were further detected by flow cytometry. Compared with the phosphate buffer solution(PBS) group, the apoptosis rate and ROS production were not increased in Ag-MOF, Ag-MOF-Vanc, and PLT@Ag-MOF-Vanc groups (Fig. 4d and e). Apoptosis is a mechanism of cell death, and ROS is a key molecule in cell apoptosis and autophagy [50]. These results indicate that the synthesized material in this study does not lead to cell death by promoting apoptosis.

\section{In vitro antibacterial effect of Ag-MOF-Vanc}

In this study, the antibacterial effect of the newly synthesized Ag-MOF on three common clinical strains (Escherichia coli ATCC25922, Pseudomonas aeruginosa ATCC27853, and S. aureus ATCC25923) was investigated by disc method. The results of antibacterial zone showed that Ag-MOF had a good inhibitory effect on the growth of the three strains (Fig. 5a and b). For MRSA (ATCC25923), the minimum inhibitory concentration (MIC) of Ag-MOF was $8 \mu \mathrm{g} / \mathrm{mL}$ (Fig. 5c). The antibacterial effects of free vancomycin, Ag-MOF-Vanc and PLT@ Ag-MOF-Vanc on MRSA were compared; the results showed that the antibacterial zones of Ag-MOF-Vanc and PLT@Ag-MOF-Vanc were larger than that of free vancomycin at different concentrations (Fig. $5 \mathrm{~d}$ and e). The MIC of free vancomycin was $2 \mu \mathrm{g} / \mathrm{mL}$; in contrast, the MIC of Ag-MOF-Vanc was $1 \mu \mathrm{g} / \mathrm{mL}$, and the MIC of PLT@Ag-MOF-Vanc was $0.5 \mu \mathrm{g} / \mathrm{mL}$ (Fig. 5f), which was much lower than that of free vancomycin. Then, we co-incubated MRSA with $0.5 \mu \mathrm{g} / \mathrm{mL}$ free vancomycin, Ag-MOF-vanc or PLT@Ag-MOF-Vanc, and colony forming unit (CFU) of bacteria was detected at $0,4,8,16$ and $24 \mathrm{~h}$, respectively. The results showed that although the growth of MRSA in free vancomycin and Ag-MOFvanc groups were lower than that in PBS group, the bacteriostatic effect was not significant, while the bacterial growth in PLT@Ag-MOF-Vanc group was significantly inhibited (Fig. 5g). The above results indicated that AgMOF could enhance the antibacterial effect of vancomycin against MRSA and reduce the dosage of vancomycin.

MRSA was exposed to different concentrations of drugs or for different periods of time, then stained with the Live/Dead backlight bacterial viability kit, and the images were measured with CLSM. When MRSA was exposed to different concentrations $(0,10,20,40 \mu \mathrm{g} /$ $\mathrm{mL}$ ) of Vanc, Ag-MOF-Vanc and PLT@Ag-MOF-Vanc for $1 \mathrm{~h}$, the number of dead bacteria increased in a dose-dependent manner, suggesting that the antibacterial activity of PLT@Ag-MOF-Vanc was concentrationdependent (Fig. 5h). MRSA was treated with $1 \mu \mathrm{g} / \mathrm{mL}$ vancomycin, Ag-MOF-Vanc and PLT@ Ag-MOF-Vanc. The permeability of the bacteria increased with prolonged time, suggesting the time dependence of PLT@ Ag-MOF-Vanc (Fig. 5i).

\section{Antibacterial mechanism of PLT@Ag-MOF-Vanc}

A series of studies were conducted to explore the antibacterial mechanism of PLT@Ag-MOF-Vanc. The first step forPLT@Ag-MOF-Vanc to exert its antibacterial effect is to target MRSA with the assistance of PLTm. In order to clarify the interaction between PLT@Ag-MOF-Vanc and MRSA, Ag-MOF-Vanc and PLT@Ag-MOF-Vanc at a certain concentration were co-incubated with MRSA for $3 \mathrm{~h}$ and observed by Scanning electron microscopy (SEM). Figure 6a shows that the surface of MRSA was relatively smooth when exposed to Ag-MOF-Vanc, while a large number of nanoparticles were attached to the surface after the exposure to PLT@Ag-MOF-Vanc. This indicated that PLTm promoted the binding of PLT@AgMOF-Vanc to MRSA and had a certain targeting effect. In order to observe the binding of the nanomaterials to bacteria more clearly, we co-incubated Cy5-labeled AgMOF-Vanc or Cy5-labeled PLT@Ag-MOF-Vanc with MRSA which were stained with DMAO, and observed with CLSM. Figure 6b showed the binding of PLT@AgMOF-Vanc to cultured $S$. aureus in vitro, in contrast to Ag-MOF-Vanc without bacterial binding.

To evaluate the effect of PLT@Ag-MOF-Vanc on bacterial metabolism, intracellular ATP levels were measured. The results showed that PLT@Ag-MOF-Vanc led to a significant decrease in ATP level, which was significantly greater than that caused by Ag-MOF and vancomycin (Fig. 6c). The decrease in ATP levels may be attributed to the inactivation of F-type ATP synthase (F-ATPase) (Fig. 6d). The functions of F-ATPase include 


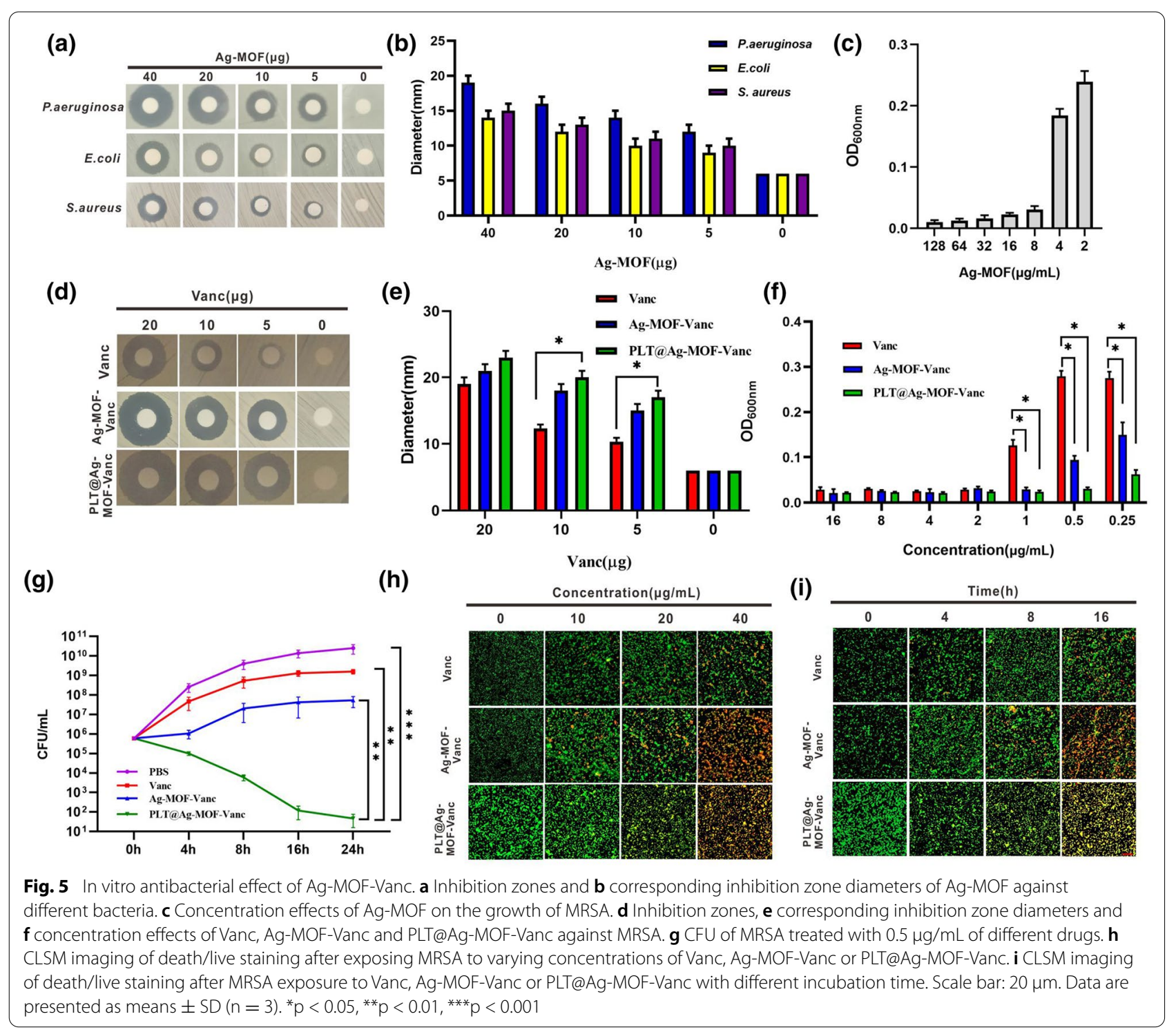

catalyzing the synthesis of ATP in the last step of oxidative phosphorylation, working in reverse as an ATPase to produce the transmembrane proton electrochemical gradient required for molecular transport [51]. PLT@ Ag-MOF-Vanc could significantly decrease the activity of F-ATPase. F-ATPase is widely expressed in mammalian cells, but as shown in Fig. 4c, Ag-MOF did not affect the cell viability. It can be indicated that Ag-MOF showed selective toxicity to bacteria but not to mammalian cells.
This selectivity may be due to the fact that Ag-MOF is difficult to affect the respiratory chain in mammalian mitochondria, which need to overcome several barriers including the escape from lysosomes, targeting mitochondria, and entry into of mitochondria through the membrane $[52,53]$.

The death of bacteria exposed to nanoparticles can be attributed to the disruption of energy production caused by the decoupling of oxidized phosphate in the cellular

(See figure on next page.)

Fig. 6 Antibacterial mechanism of PLT@Ag-MOF-Vanc. a SEM images of MRSA incubated with Ag-MOF-Vanc or PLT@ Ag-MOF-Vanc. Scale bar: $1 \mu \mathrm{m} \mathbf{b}$ CLSM images of MRSA incubated with Cy5-labeled Ag-MOF-Vanc and Cy5-labeled PLT@Ag-MOF-Vanc. Scale bar: $2 \mu \mathrm{m}$. c Intracellular ATP level, d F-type ATPase activity, (3) DCFH-DA fluorescence counts, and e MDA contents of MRSA treated with Ag-MOF, vancomycin, Ag-MOF-Vanc, or PLT@ Ag-MOF-Vanc. g Crystal violet staining, h XTT dyeing, and i CLSM imagaes of MRSA biofilm. Scale bar: $20 \mu$ m. Data are presented as means $\pm S D(n=3) .{ }^{*} p<0.05$ 
(a)

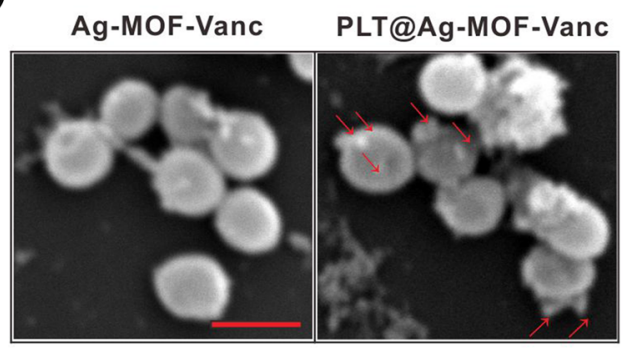

(b)

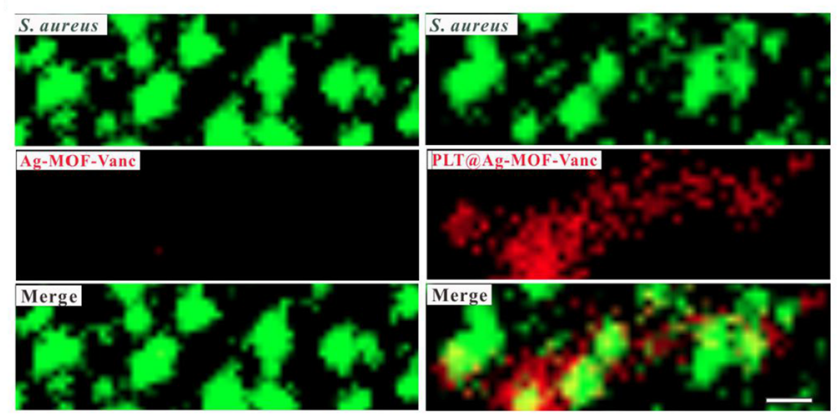

(c)

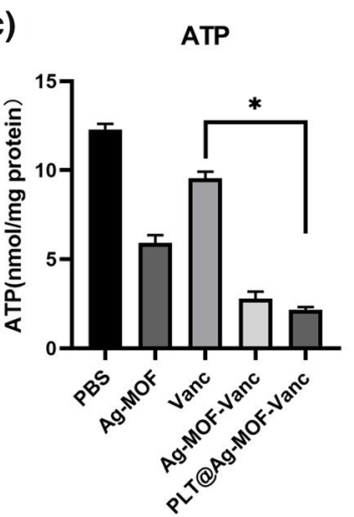

(e)

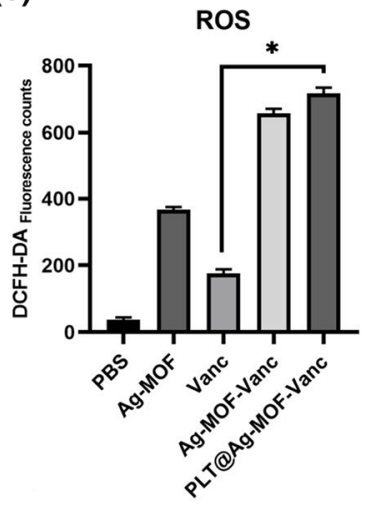

(g)

(d)

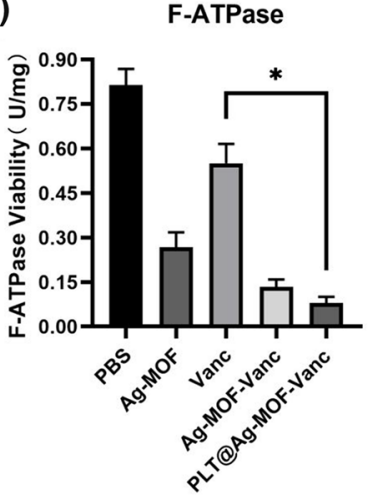

(f)

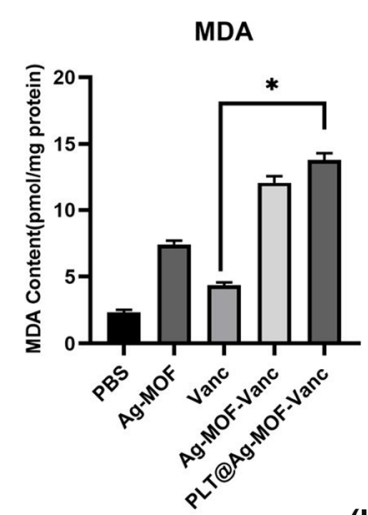

crystal violet
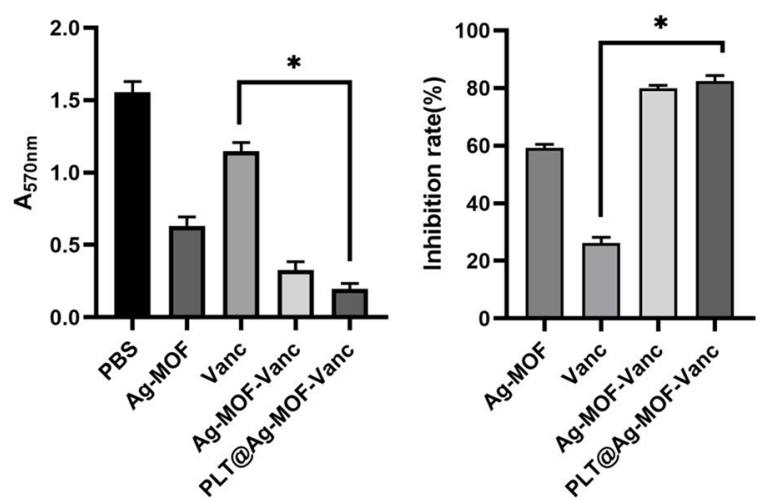

(h)
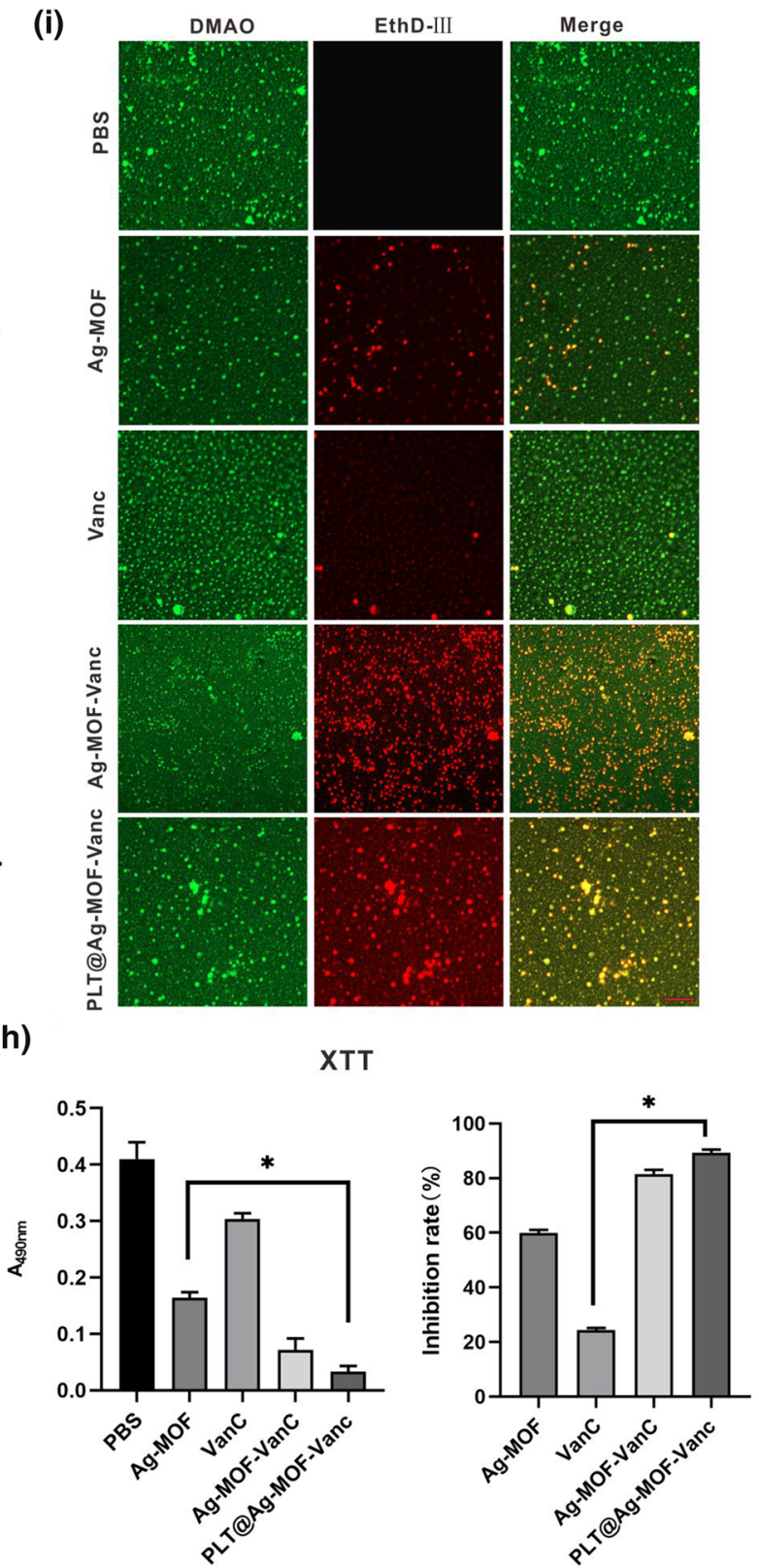
respiratory chain, the interference in membrane permeability, and the loss of enzyme activity involved in key metabolic pathways; among them, the excessive ROS production by cells is the most effective component for triggering bacterial cell death [54]. Therefore, it is very important to study the effect of nanoparticles on the formation of ROS in bacterial cells. We used 2,7-dichlorodihydrofluorescein diacetate (DCFH-DA) method to quantitatively detect ROS. Figure 6e shows the ROS production level of MRSA after the treatment with AgMOF, free vancomycin, Ag-MOF-Vanc, and PLT@AgMOF-Vanc. Compared with the control group and the free vancomycin group, Ag-MOF treatment significantly increased ROS levels. The variation was more obvious in the bacteria treated with Ag-MOF-Vanc and PLT@AgMOF-Vanc. High ROS levels were observed in PLT@ Ag-MOF-Vanc-treated bacteria, indicating that PLT@ Ag-MOF-Vanc effectively bound to the bacterial surface, thus releasing a high proportion of silver ions in the target cells.

One of the main consequences of intracellular ROS accumulation is the damage to the membrane integrity caused by the gradual establishment of oxidative stress. In addition, nanoparticles can also cause physical damage to the cell membrane. Therefore, we continued to use malondialdehyde (MDA) method to detect cell lipid peroxidation to determine the degree of membrane damage. There were significant differences in MDA content among bacteria in different treatment groups (Fig. 6f). Compared with free vancomycin, the content of MDA in PLT@Ag-MOF-Vanc-treated cells increased significantly. These results suggested that the interaction between AgMOF and bacterial surface increased the degree of bacterial damage.

One of the reasons for drug resistance and poor therapeutic effect of antibiotics is the generation of biofilms. Because of their high permeability, nanoparticles can penetrate thick biofilms. We speculated that Ag-MOFVanc may have a good inhibitory and scavenging effect on S. aureus biofilm. During the early- and mid-stages during biofilm formation, two assays, crystal violet staining and the 2,3-bis (2-methoxy-4-nitro-5-sulfophenyl)-2 H-tetrazolium-5-carbox-anilide (XTT) assay, are regarded as crucial experimental tools. The results of crystal violet staining and XTT staining after treating the MRSA biofilm with different drugs showed that PLT@Ag-MOF-Vanc effectively destroyed the biofilm formed by MRSA, and the effect was obviously better than that of Ag-MOF and vancomycin alone (Fig. $6 \mathrm{~g}$ and $\mathrm{h}$ ). Bacterial biofilm formation was also confirmed by using LIVE/DEAD backlight bacterial viability staining and observed by CLSM. As shown in Fig. 6i, after 24 h of treatment with PLT@Ag-MOF-Vanc, there were maximum fluorescence intensities of red fluorescence, which reflected that the PLT@Ag-MOF-Vanc killed biofilms of MRSA. The results obtained by CLSM were identical to those obtained by crystal violet and XTT analysis.

In conclusion, PLT@Ag-MOF-Vanc could kill MRSA through a comprehensive physical and chemical mechanism, including targeting MRSA via PLTm; interfering with the intracellular metabolism of bacteria; catalytic production of ROS; damage to cell membrane integrity; and inhibiting the formation of biofilm.

\section{Distribution of intravenously injected PLT@ Ag-MOF-Vanc}

To demonstrate that PLT@Ag-MOF-Vanc can target the MRSA-infected sites in vivo, biodistribution in MRSA pneumonia model mice was evaluated by Small Animal In Vivo Imaging at 6, 24, and $48 \mathrm{~h}$ after Ag-MOF-Vanc and PLT@Ag-MOF-Vanc tail vein injection. As shown in Fig. 7a, there is a significant difference in the distribution of Ag-MOF-Vanc and PLT@Ag-MOF-Vanc. PLT@ Ag-MOF-Vanc was significantly accumulated in lungs within $48 \mathrm{~h}$ of administration and seldom accumulated in brain, heart, liver, spleen and kidney, while Ag-MOFVanc rarely aggregated at the infected site within $48 \mathrm{~h}$ after injection. In the Ag-MOF-Vanc group, there were obvious signals in the bladder, which might attribute to the accumulation of Ag-MOF-Vanc in the bladder after metabolism by the kidney and then excreted in the urine. $48 \mathrm{~h}$ after injection, the mice were killed by cervical dislocation, and fluorescence imaging of the lung, brain, liver, heart, spleen and kidney was performed ex vivo (Fig. 7b and c). There was a small amount of Ag-MOF-Vanc aggregation in the lung, liver, and spleen; the accumulation of PLT@Ag-MOF-Vanc in the lung was much higher than that of Ag-MOF-Vanc. The above results indicated that PLT@Ag-MOF-Vanc has a good targeting effect on the MRSA-infected sites in vivo.

\section{Anti-infection effect of PLT@Ag-MOF-Vanc in vivo}

The present study further evaluated the anti-infection effect of PLT@Ag-MOF-Vanc in vivo in the MRSA pneumonia model of Kunming mice. The infected mice were divided into the following five groups: normal saline group, Ag-MOF group, vancomycin group, Ag-MOFVanc group, and PLT@Ag-MOF-Vanc group. We carried out the anti-infection experiments in vivo according to the Fig. 8a. After establishing the model, the corresponding drugs were injected daily. According to the vancomycin concentration, the dosage was $2 \mathrm{mg} / \mathrm{kg}$ every day for vancomycin group, Ag-MOF-Vanc group, and PLT@Ag-MOF-Vanc group. The dosage of Ag-MOF group was the same as the concentration of Ag-MOF contained in Ag-MOF-Vanc and PLT@Ag-MOF-Vanc groups. Every day one mouse was taken from each group 


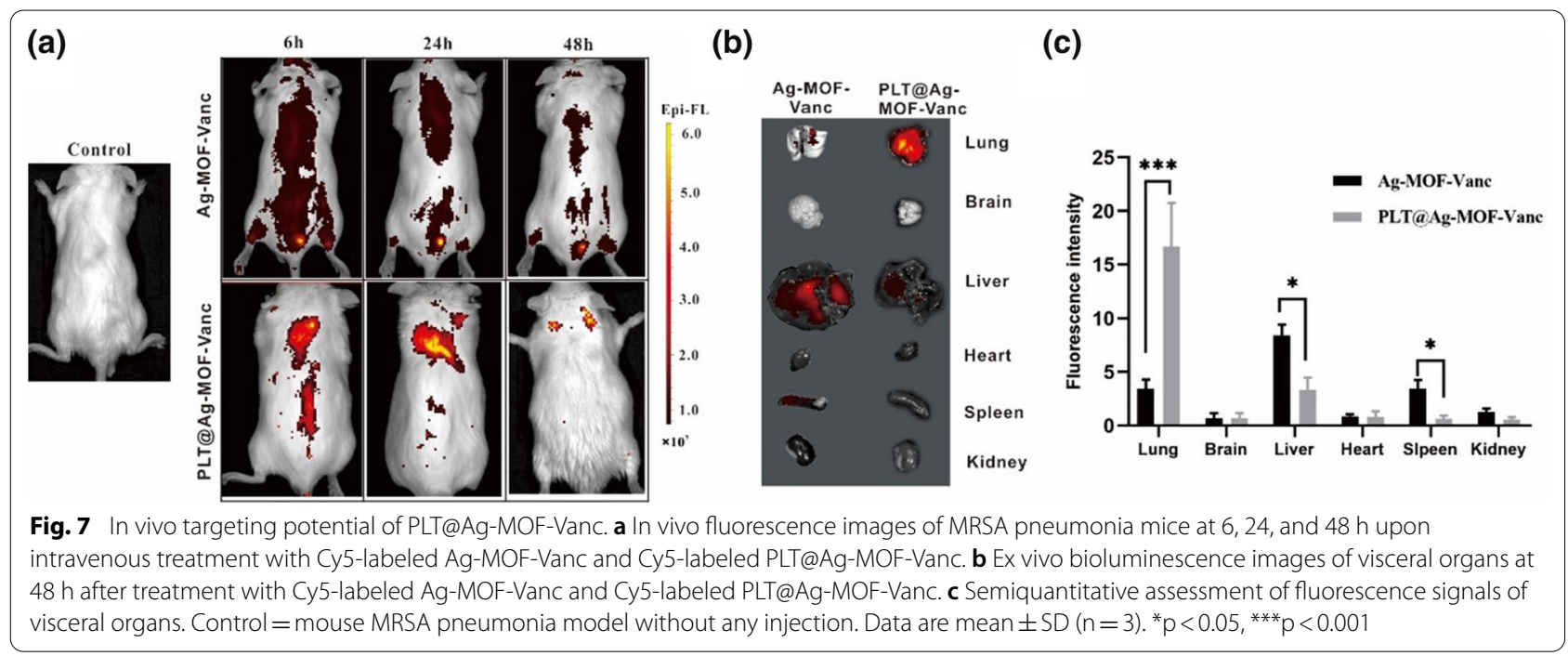

for Hematoxylin-eosin(HE) staining so as to observe the alveolar structure and integrity of ciliated endothelial cells, inflammation, necrosis, and infiltration by inflammatory cells (macrophages) in the alveoli. The results showed significantly better improvement rate of the lung condition in PLT@Ag-MOF-Vanc group compared with other groups, and the alveoli recovered from the third day of the treatment, with no obvious inflammatory cell infiltration (Fig. 8b). Four days after the treatment, the levels of inflammatory cytokines interleukin-6 (IL-6) and tumor necrosis factor- $\alpha$ (TNF- $\alpha$ ) in the lung tissue of mice were examined by immunohistochemical staining; the results showed that the expression levels of IL- 6 and TNF- $\alpha$ in the normal saline group, Ag-MOF group, vancomycin group, and Ag-MOF-Vanc group were still significantly higher compared with the normal control mice, while those in PLT@Ag-MOF-Vanc group almost returned to normal control level (Fig. 8c). In addition, blood was taken for hematological tests; the results showed that the white blood cell (WBC), neutrophil (NEU) count and inflammatory marker $\mathrm{C}$-reactive protein (CRP) level were significantly reduced in the PLT@Ag-MOF-Vanc group (Fig. 8d). The levels of IL-6 and TNF- $\alpha$ in blood were examined by enzyme-linked immuno sorbent assay (ELISA); it was shown that the level of inflammatory cytokines significantly decreased in the PLT@Ag-MOFVanc group (Fig. 8d). The bacterial count in alveolar lavage fluid of different treatment groups also showed that the number of residual bacteria was the lowest after the treatment with PLT@Ag-MOF-Vanc (Fig. 8e).

We reprepared a group of mice infected with MRSA pneumonia and randomized them to receive corresponding treatment to observe the survival rate of each group. MRSA killed all mice treated with saline, and different numbers of mice died within 7 days in the AgMOF group, vancomycin group and Ag-MOF-Vanc group, but the survival rate of mice in the PLT@AgMOF-Vanc group was $100 \%$ (Fig. 8f). In the PLT@AgMOF-Vanc group, the concentration of vancomycin was $2 \mathrm{mg} / \mathrm{kg}$, while the survival rate in the free vancomycin group $(2 \mathrm{mg} / \mathrm{kg})$ was only $50 \%$, indicating that under the same dose of vancomycin, the therapeutic effect of PLT@Ag-MOF-Vanc was better than that of free vancomycin. According to the literature [39], in the treatment of mouse pneumonia model, vancomycin should reach at least $15 \mathrm{mg} / \mathrm{kg}$, and the survival rate can reach $100 \%$, indicating that PLT@Ag-MOF-Vanc involved in this study can effectively reduce the dose of vancomycin.

All the above results show that PLT@Ag-MOF-Vanc has a good anti-infective effect in mouse MRSA pneumonia model, which is significantly superior to free vancomycin and Ag-MOF alone or uncoated Ag-MOFVanc, indicating that Ag-MOF and vancomycin have a synergistic anti-infective effect. Meanwhile, after encapsulation with PLTm, PLT@Ag-MOF-Vanc could be targeted and transported to the MRSA-infected site, thus further strengthening the anti-infective effect of PLT@ Ag-MOF-Vanc.

\section{In vivo toxicity assessment of PLT@Ag-MOF-Vanc}

In order to evaluate the potential toxicity of PLT@AgMOF-Vanc, healthy Kunming mice were randomized into five groups and injected with $100 \mu \mathrm{L}$ of normal saline, Ag-MOF, vancomycin, Ag-MOF-Vanc and PLT@ Ag-MOF-Vanc. According to the vancomycin concentration, the dosage was $2 \mathrm{mg} / \mathrm{kg}$ for vancomycin group, Ag-MOF-Vanc group, and PLT@Ag-MOF-Vanc group. The dosage of Ag-MOF group was the same as 
(a)

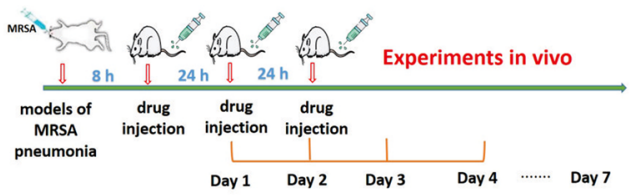

(b)

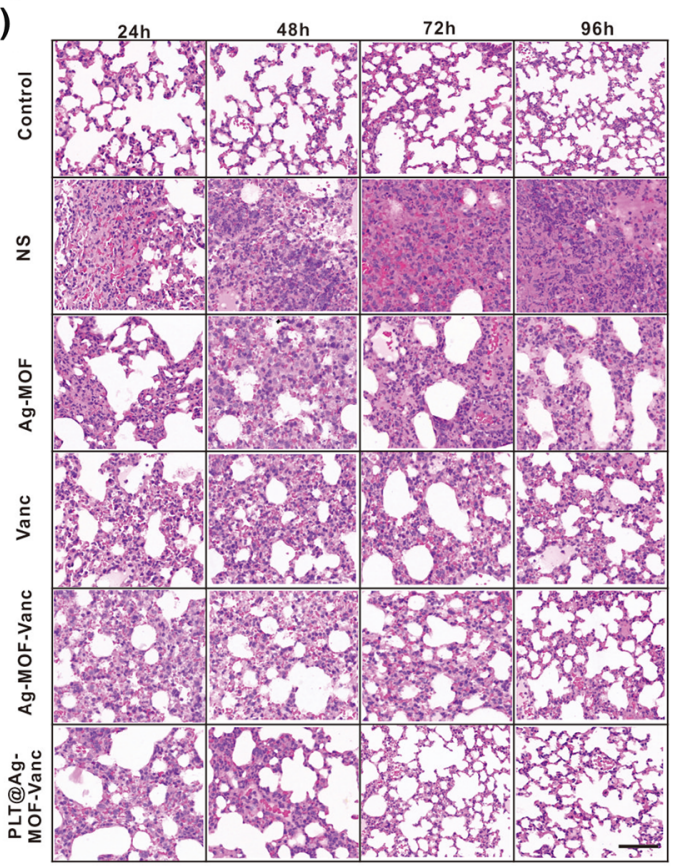

(d)
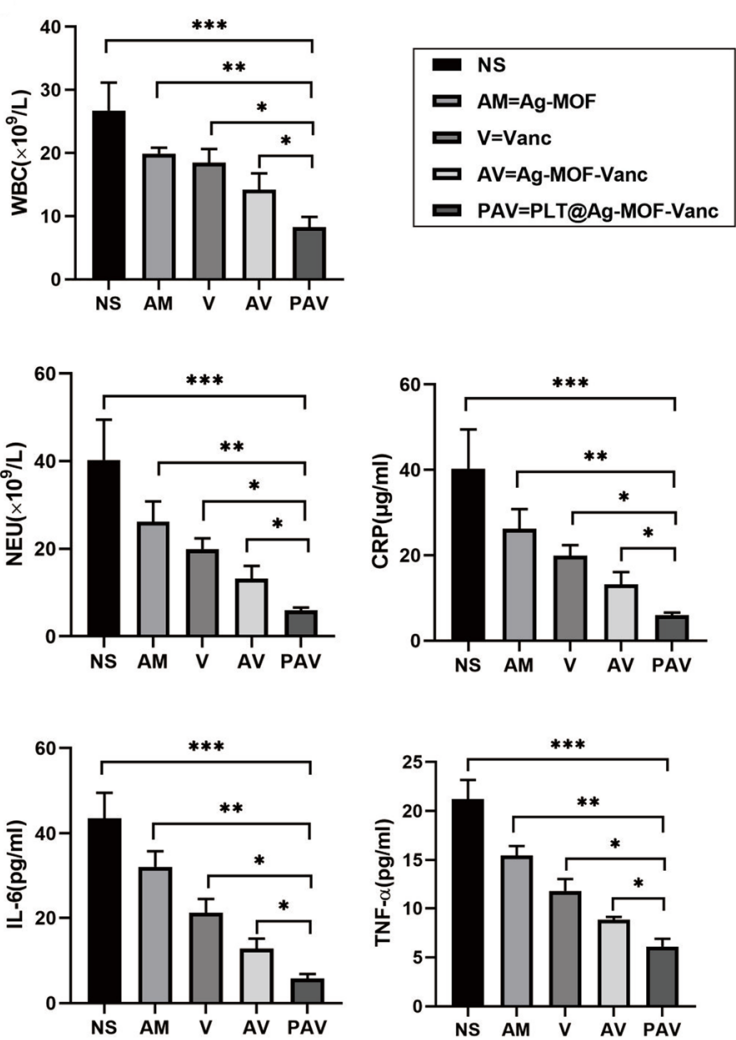

(c)

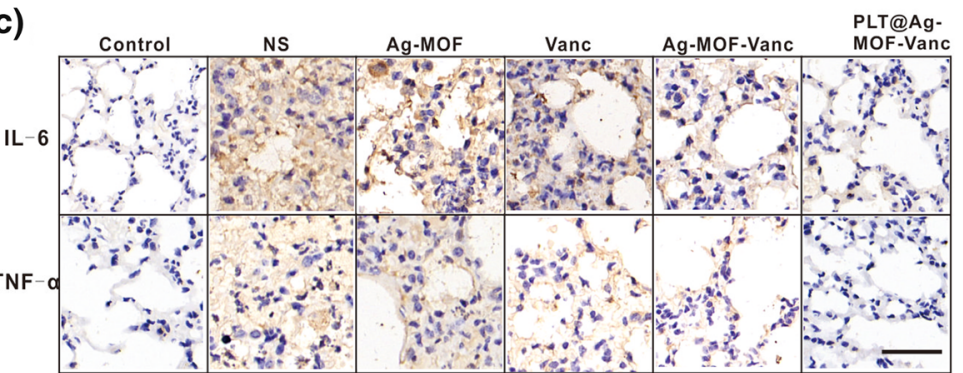

(f)

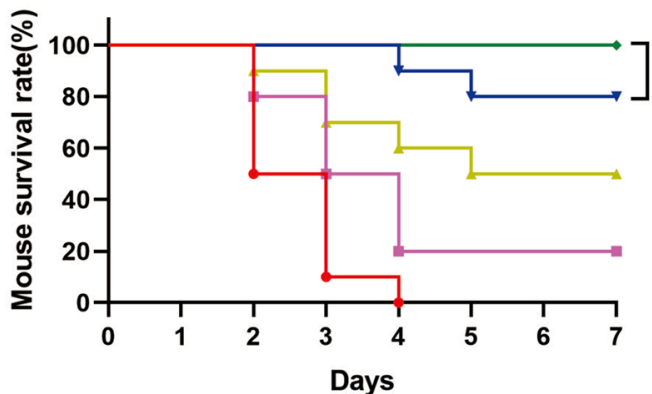

(e)

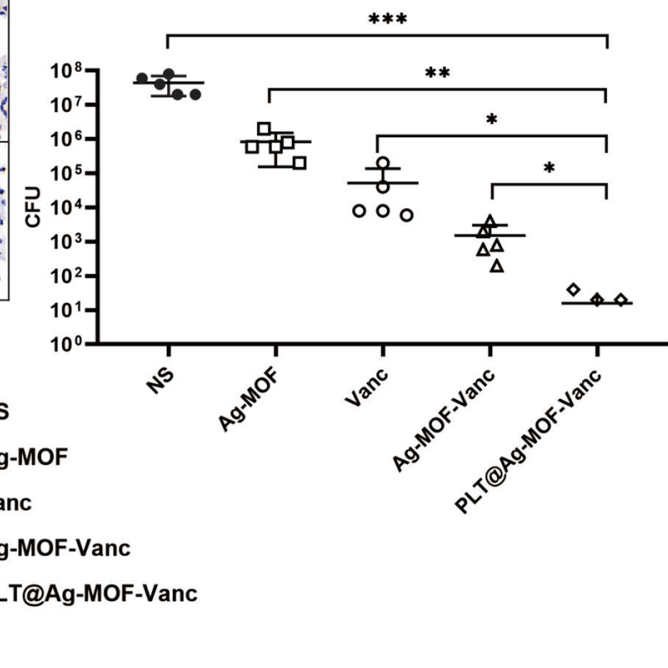

Fig. 8 Anti-infection effect of PLT@Ag-MOF-Vanc in vivo. a Schematic diagram of in vivo anti-infection experimental design. $\mathbf{b} \mathrm{HE}$ staining images of lung tissues. $\mathbf{c}$ Immunohistochemical staining of IL-6 and TNF-a in lung tissues. $\mathbf{d}$ Inflammation-associated cell counts and cytokine levels in the blood. Data are presented as means $\pm S D(n=5)$. e Bacterial count in alveolar lavage fluid $(n=5)$. f Survival rates in the mouse MRSA pneumonia model after tail-vein injection of different drugs $(n=10)$. Control $=$ normal healthy mice. Scale bar: $100 \mu m$. ${ }^{*} p<0.05,{ }^{* *} p<0.01,{ }^{* * *} p<0.001$ 


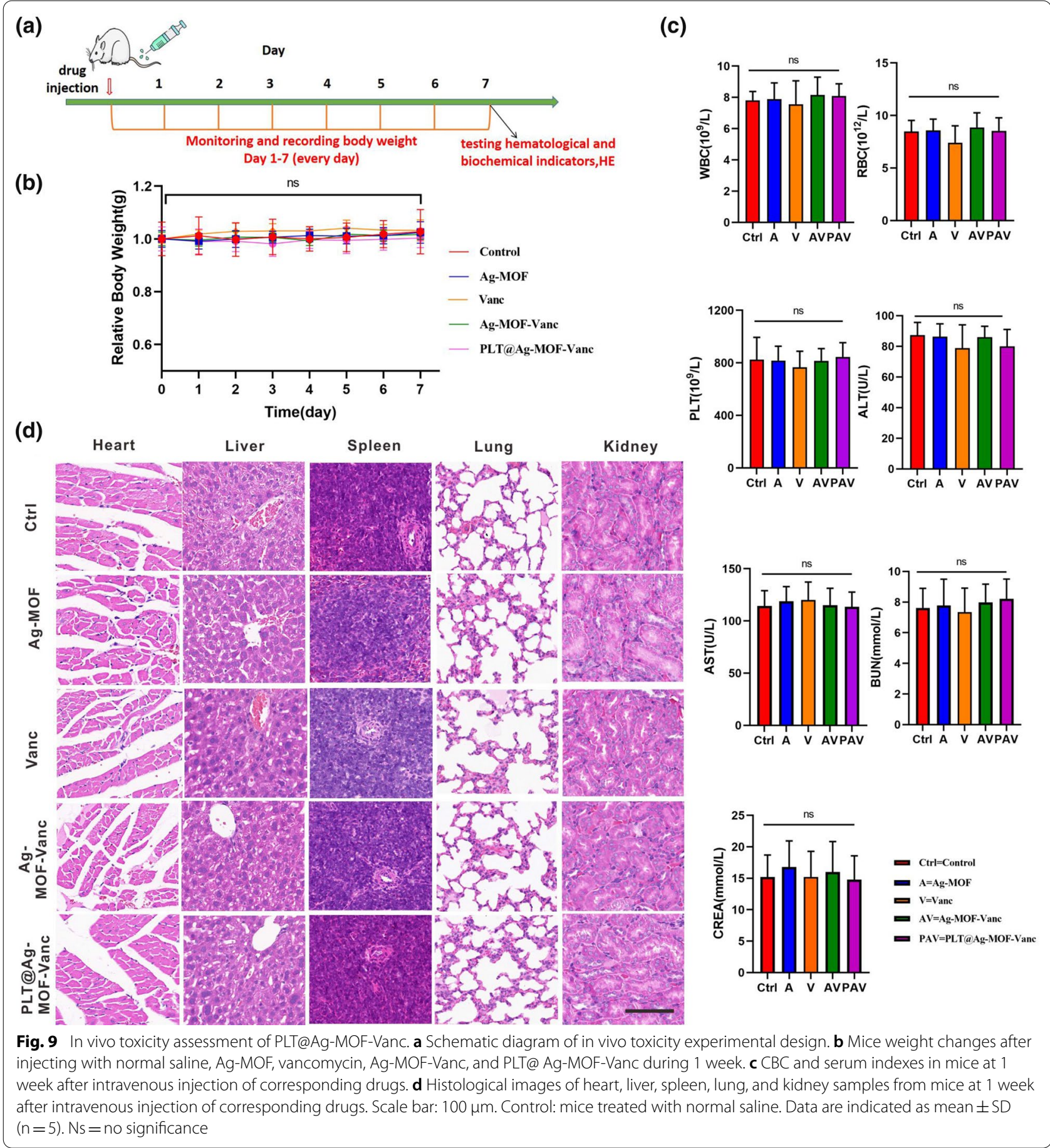

the concentration of Ag-MOF contained in Ag-MOFVanc and PLT@Ag-MOF-Vanc groups. As shown in the Fig. 9a, we designed the toxicity experiments in vivo. We recorded the changes in mouse body weights every day for 1 week after tail vein drug injection, and found no significant difference in body weight among the five groups (Fig. 9b). Hematological indicators of normal mice were measured 1 week after the tail vein drug injection. We measured complete blood counts (red blood cell (RBC), WBC, platelet (PLT)), liver function indicators (alanine transaminase (ALT), aspartate aminotransferase (AST)), and renal function indicators (blood urea nitrogen (BUN), and creatinine (CREA)). There was no significant difference in any of these indicators among different 
groups (Fig. 9c), indicating that PLT@Ag-MOF-Vanc had no significant effect on the production of red blood cells, white blood cells, and platelets in blood, and it had no obvious hepatorenal toxicity.

To further clarify the in vivo toxicity of PLT@Ag-MOFVanc, we used HE staining to evaluate the pathological changes in major organs (heart, liver, spleen, lung, and kidney) and we didn't observe any arrested necrosis, fibrosis, histological abnormalities, and inflammatory lesions among the five groups (Fig. 9d). The results demonstrated that PLT@Ag-MOF-Vanc did not cause significant damage to the heart, liver, spleen, lung, and kidney; it had low toxicity in vivo and good biocompatibility.

\section{Conclusions}

In this study, we designed and synthesized a PLTmcamouflaged nano-drug delivery system (PLT@AgMOF-Vanc). The drug release and silver ion release of PLT@Ag-MOF-Vanc were shown to be pH-responsive, which can prevent the delivery system from prereleasing drugs in the circulatory system. The platelet membrane on PLT@Ag-MOF-Vanc effectively covers the internal nano-core, reduces the recognition and clearance of reticuloendothelial system, and has good biocompatibility. Ag-MOF-Vanc showed good antibacterial activity against common clinical strains in vitro, significantly better than free vancomycin. In the MRSA pneumonia model, PLT@Ag-MOF-Vanc targeted the MRSA-infected sites. Combining the antibacterial ability of Ag-MOF with vancomycin, the growth of MRSA was inhibited and the effect was better than when using vancomycin alone; in that way, when the same dosage of vancomycin was used, the therapeutic effect of PLT@Ag-MOF-Vanc was significantly better than that of free vancomycin, and no obvious toxicity was observed.PLT@Ag-MOF-Vanc is a novel and effective targeted drug delivery system that can be used for safe and effective anti-infection therapy.

\section{Materials and methods \\ Materials}

Silver nitrate and 2-methylimidazole were purchased from Aladdin (China). Cy5 and Hoechst 33,342 were provided by Yeasen Biotechnology (China) Co., Ltd. Dialysis membrane $(2 \mathrm{kD})$ was purchased from SolarBio (China). Annexin V-FITC/PI Apoptosis assay Kit and ROS assay Kit were provided by Beyotime Biotechnology (China). Cell Counting Kit-8 (CCK-8) was manufactured by Dojindo Laboratories, Japan. Primary antibodies against TNF- $\alpha$ and IL- 6 monoclonal antibodies were purchased from Proteintech (China). Viability/Cytotoxicity Assay for Bacteria Live and Dead Cells was purchased from US Everbright Inc. (USA).

\section{Cells and mice}

HUVECs, MLE12 and RAW264.7 cells were bought from the Advanced Research Center, Central South University; they were cultured in RPMI-1640 medium supplemented with $10 \%$ fetal bovine serum. The cells were kept at $37{ }^{\circ} \mathrm{C}$ in $5 \% \mathrm{CO}_{2}$ at atmospheric pressure. Kunming mice (male, 8 weeks old) were bought from Hunan SJA Laboratory Animal Co., Ltd.

\section{Synthesis of Ag-MOF}

First, $1 \mathrm{~g}$ 2-methylimidazole was dissolved in $10 \mathrm{~mL}$ $\mathrm{ddH}_{2} \mathrm{O} ; 57 \mathrm{mg}$ silver nitrate was dissolved in $2 \mathrm{~mL}$ $\mathrm{ddH}_{2} \mathrm{O}$; then, the silver nitrate solution was dropped into the 2-methylimidazole aqueous solution. The solution was placed in a polyethylene reaction pot at $120{ }^{\circ} \mathrm{C}$ for 15 min, washed 2-3 times using $\mathrm{dd}_{2} \mathrm{O}$, and centrifuged at $14,000 \mathrm{rpm}$ for $10 \mathrm{~min}$.

\section{Synthesis of Ag-MOF-Vanc}

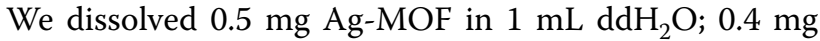
vancomycin was added, stirred overnight at room temperature by magnetic force, and Ag-MOF-Vanc was obtained by centrifugation.

\section{Preparation of PLTm vesicles}

The whole blood of male Kunming mice was used and placed in tubes containing heparin. Platelets were isolated from whole blood by centrifugation and washing. PLTm were extracted by repeated freeze-thaw extraction, suspended in PBS, and then subjected to ultrasonic treatment $(2 \mathrm{~min}, 42 \mathrm{kHz}, 100 \mathrm{~W})$ to produce PLTm vesicles.

\section{Construction of PLT@Ag-MOF-Vanc}

PLTm vesicles were fused with equal volume of Ag-MOFVanc by ultrasound ( $5 \mathrm{~min}, 42 \mathrm{kHz}, 100 \mathrm{~W}$ ). The sample was filtered 20 times using a porous syringe filter with a membrane pore diameter of $200 \mathrm{~nm}$ and centrifuged (2500 rpm, $10 \mathrm{~min}$ ) to remove the excess PLTm to separatePLT@Ag-MOF-Vanc.

\section{Characterization}

Ag-MOF, PLTm and PLT@Ag-MOF were observed through TEM with Tecnai G2 Spirit TEM (FEI, USA) to confirm that the PLTm was wrapped on the surface of Ag-MOFs. Elemental mapping analysis was performed by FEI Talos F200x with an integrated Super-X EDS system (FEI, USA). Zetasizer Nano ZS (Malvern Nano Series, Malvern, UK) was used to evaluate the particle size distribution and zeta potential. The PLTm proteins were identified by SDS-PAGE. The absorption spectra of AgMOF-Vanc were obtained using UV-Vis (Scandrop, Analytik Jena, Germany). The molecular functional groups of 
Ag-MOF-Vanc were studied by Fourier-transform infrared spectroscopy (FTIR).

\section{Determination of EE and LE}

We dissolved $0.5 \mathrm{mg}$ Ag-MOF in $1 \mathrm{~mL} \mathrm{ddH}_{2} \mathrm{O}$, and $0.4 \mathrm{mg}$ vancomycin was added and stirred overnight by magnetic stirring at room temperature. Ag-MOF-Vanc was obtained by centrifugation. The maximum absorbance of vancomycin was determined by UV-vis spectrophotometry. The standard concentration gradient was set and the standard curve of concentration and absorbance was established; then, we calculated the uncombined vancomycin in the supernatant. The calculation formulas for LE and EE were as follows:

$$
\begin{gathered}
\mathrm{EE}=\text { (quality of drugs contained on nano-carrier } / \\
\text { total amount of drugs used }) \times 100 \%,
\end{gathered}
$$

$$
\begin{aligned}
\mathrm{LE}= & \text { (mass of drug contained on nano-carrier } / \\
& \text { mass of nano-carrier }) \times 100 \% .
\end{aligned}
$$

\section{Release of vancomycin and silver ion}

In vitro drug release experiments were carried out under pH 7.4 and pH 6.5 to observe whether PLT@Ag-MOFVanc could release vancomycin and silver ion more easily under acidic environment. Next, 2 mL PLT@Ag-MOFVanc was put into a $2 \mathrm{kD}$ dialysis bag and immersed in 20 $\mathrm{mL}$ PBS solution at $\mathrm{pH} 7.4$ and $\mathrm{pH} 6.5$, seperately. The absorbance of vancomycin in dialysate was determined at $280 \mathrm{~nm}$. Cumulative release of vancomycin was based on the standard curve. The concentration of $\mathrm{Ag}^{+}$in dialysate was determined by a direct-reading inductively coupled plasma emission spectrometer (Spectro Blue, Spectro, Germany).

\section{Biocompatibility of PLT@Ag-MOF-Vanc}

The biocompatibility of PLT@Ag-MOF-Vanc was evaluated by hemolysis rate and macrophage phagocytosis test.PLT@Ag-MOF-Vanc specimens of different concentrations $(5-160 \mu \mathrm{g} / \mathrm{mL})$ were mixed with $5 \%$ Kunming mice erythrocyte suspension, incubated at $37{ }^{\circ} \mathrm{C}$ for $2 \mathrm{~h}$, and centrifuged at $3500 \mathrm{rpm}$ for $5 \mathrm{~min}$. The absorbance was obtained at $545 \mathrm{~nm}$ using ultra-pure water and normal saline as positive and negative controls, respectively. The evaluation formula of hemolysis rate was as follows: hemolysis rate $(\%)=$ (absorbance of experimental sample - absorbance of negative control)/(absorbance of positive control-absorbance of negative control) $\times 100$. To determine the immune escape ability of PLT@Ag-MOF-Vanc, RAW264.7 cells were inoculated in a 6 -well plate (about $3 \times 10^{5}$ cells/ well) and incubated for 24 h. PLT@Ag-MOF-Vanc and Ag-MOF-Vanc were added and incubated for $24 \mathrm{~h}$ and then stained with Hoechst 33,342. The phagocytosis and fluorescence signals of macrophages against PLT@ Ag-MOF-Vanc were obtained under CLSM (Zeiss LSM 800, Germany).

The cytotoxicity of PLT@Ag-MOF-Vanc was evaluated using HUVECs and MLE12 cells. The cells were inoculated in 96-well plate $\left(2 \times 10^{3}\right.$ cells/well $)$ and incubated for $24 \mathrm{~h}$. The concentrations of Ag-MOF-Vanc and PLT@Ag-MOF-Vanc were calculated with the concentration of vancomycin as $20.0 \mu \mathrm{g} / \mathrm{mL}$. The dosage of $\mathrm{Ag}-\mathrm{MOF}$ group was the same as the concentration of Ag-MOF contained in Ag-MOF-Vanc and PLT@AgMOF-Vanc groups. After incubation for $48 \mathrm{~h}, \mathrm{CCK}-8$ solution $(10 \mu \mathrm{L})$ was added to each well for $3 \mathrm{~h}$, and the absorbance was measured at $450 \mathrm{~nm}$.

Cell apoptosis was detected by Annexin V-FITC/PI apoptosis detection kit. In brief, the cells were inoculated at $10^{6}$ cells/bottle in T25 culture containers and incubated for $24 \mathrm{~h}$ until the cells adhered to the wall. Ag-MOF, Ag-MOF-Vanc and PLT@Ag-MOF-Vanc were added for further incubation for $48 \mathrm{~h}$. Apoptosis was detected by flow cytometry as per standard procedures of the Annexin V-FITC/PI Apoptosis Detection Kit.

The production of ROS was detected. The cells were inoculated at $10^{6}$ cells/bottle in T25 culture containers and incubated for $24 \mathrm{~h}$ until the cells adhered to the wall. Ag-MOF, Ag-MOF-Vanc and PLT@Ag-MOF-Vanc were added for further incubation for $48 \mathrm{~h}$. After additional incubation for $48 \mathrm{~h}$, the intracellular ROS were analyzed by flow cytometry in accordance with the standard operation of the ROS detection kit.

\section{In vitro antibacterial experiment Disc diffusion method}

After single bacterial colony was obtained, the concentration of the bacterial solution was adjusted to $0.5 \mathrm{MCF}$ with normal saline, and the bacterial solution was evenly spread on Mueller-Hinton ( $\mathrm{MH}$ ) medium. After placing a paper on the staining medium, different contents of the Ag-MOF, vancomycin, or Ag-MOF-Vanc were injected into the paper. After incubation for 1 day, the inhibition zone was observed.

\section{Dilution method}

First, we took one to two MRSA colonies and added them to PBS solution for dilution. Then, we added them to the Luria-Bertani (LB) culture medium so that the final concentration of the bacteria was $5 \times 10^{5} \mathrm{CFU} / \mathrm{mL}$. We added a higher concentration of the drug and $200 \mu \mathrm{L}$ 
bacterial solution mentioned above to the first row of a 96-well plate. We added $100 \mu \mathrm{L}$ bacterial solution to the remaining row of the 96-well plate without antibiotics. Then, we evenly mixed the bacterial solution containing antibiotics in the first row and took out $100 \mu \mathrm{L}$ and added into the second row. We mixed the solution in the second row evenly, then extracted $100 \mu \mathrm{L}$ to the third row, and so on. Then, the 96-well plate was placed in the incubator for $16-20 \mathrm{~h}$, and the absorption at $600 \mathrm{~nm}$ was read with a microplate reader. To count the colonies, the cultured bacterial solution was gradually diluted in a gradient of 10 times, dropped on the blood agar plate, and then incubated at $37{ }^{\circ} \mathrm{C}$ for $18-24 \mathrm{~h}$ for bacterial colony count.

\section{Staining of live and dead bacteria}

DMAO/EthD- III and other dyes were mixed with the bacteria solution to be tested in a certain proportion. They were mixed well and incubated in the dark at room temperature for $15 \mathrm{~min}$. Next, $5-\mu \mathrm{L}$ stained bacterial suspension droplets were placed on a glass slide with an $18-\mathrm{mm}$ square cover glass and observed by CLSM(Zeiss LSM 800, Germany). Live (green fluorescence) and dead (red fluorescence) bacteria were observed using FITC and CY3 channels, respectively.

\section{Antibacterial mechanism-related experiments Targeting ability of PLT@Ag-MOF-Vanc observed by SEM} Ag-MOF-Vanc and PLT@Ag-MOF-Vanc were mixed with bacteria for $3 \mathrm{~h}$ and fixed at room temperature with $2.5 \%$ glutaraldehyde, $2.5 \%$ paraformaldehyde, and $0.1 \mathrm{M}$ calcium carbonate buffer solution dissolved in deionized water for $2 \mathrm{~h}$. Cover slides were prepared with one drop of $1 \%$ polylysine to capture the cells. We placed one drop of the fixed medium on the cover glass and incubated with water for $5 \mathrm{~min}$ before rinsing. The sample was then dehydrated with a series of ethanol concentrations (25\%, $50 \%, 75 \%$, and $95 \%$ ) and washed three times in $100 \%$ ethanol. The sample was air-dried for about $24 \mathrm{~h}$, then coated with $\mathrm{Au}$ and Pd (7 nm thick) on the biofilm, and observed by SEM (Quanta 250FEG, USA).

\section{Targeting ability of PLT@Ag-MOF-Vanc observed by CLSM}

The bacteria were co-incubated with DMAO green fluorescent dye for $20 \mathrm{~min}$. After centrifugation, the bacteria solution was resuspended with PBS. Cy5-labeled Ag-MOF-Vanc or Cy5-labeled PLT@Ag-MOF-Vanc were mixed with bacteria for $3 \mathrm{~h}$. After washing with PBS for three times, we collected pictures with CLSM.

\section{ATP and F-ATPase}

Logarithmic growth MRSA $\left(1 \times 10^{6} \mathrm{CFU}\right)$ was collected and resuspended in PBS buffer $(\mathrm{pH}=7.4)$. Different drugs were added to the bacteria and cultured at $37{ }^{\circ} \mathrm{C}$ for $6 \mathrm{~h}$. The bacteria were divided, and the supernatant was collected after centrifugation at $1000 \mathrm{~g}$. Then, the ATP level was determined using the ATP kit (Beyotime Biotechnology, China), and the F-ATPase activity was detected by F-ATPase activity kit (GenMed Scientifics, Shanghai, China).

\section{ROS}

Logarithmic growth MRSA $\left(1 \times 10^{6}\right.$ CFU $)$ was collected and resuspended in PBS buffer $(\mathrm{pH}=7.4)$. After ROS staining, different drugs were added and incubated at $37{ }^{\circ} \mathrm{C}$ for $1 \mathrm{~h}$. Fluorescence was detected by flow cytometry.

\section{MDA}

Ag-MOF, vancomycin, Ag-MOF-Vanc, or PLT@AgMOF-Vanc were co-incubated with bacteria, centrifuged (12,000 rpm, $2 \mathrm{~min})$, resuspended in $1 \mathrm{~mL}$ of $2.5 \%$ freshly configured trichloroacetic acid (TCA), and then centrifuged $\left(12,000 \mathrm{rpm}, 20 \mathrm{~min}, 4{ }^{\circ} \mathrm{C}\right)$. The supernatant was diluted with $5 \%$ thiobarbituric acid; the same volume of TCA was added, and the mixture was heated in $100{ }^{\circ} \mathrm{C}$ water bath for $30 \mathrm{~min}$, followed by centrifuge $\left(12,000 \mathrm{rpm}, 20 \mathrm{~min}, 4{ }^{\circ} \mathrm{C}\right)$. We determined the absorbance at $532 \mathrm{~nm}$ and calculated MDA content $(\mathrm{pg} / \mathrm{mL})$ with a molar extinction coefficient of $1.56 \mathrm{nM}^{-1} \mathrm{~cm}^{-1}$.

\section{Biofilm detection \\ CLSM}

$100 \mu \mathrm{L}$ of drug culture solution was added to each well in the fluorescent confocal plate; $100 \mu$ l of overnight cultured bacteria solution was inoculated and incubated at $37{ }^{\circ} \mathrm{C}$ for $24 \mathrm{~h}$. After washing the plate for three times with PBS, we added DMAO/EthD- III dye and incubated at $37{ }^{\circ} \mathrm{C}$ in dark for $20 \mathrm{~min}$. After washing with PBS for three times and drying, we collected pictures with CLSM.

\section{Crystal violet staining}

We added $100 \mu \mathrm{l}$ of drug culture solution to each well of the 96-well plate; $100 \mu \mathrm{l}$ of overnight cultured bacteria solution was inoculated and incubated at $37{ }^{\circ} \mathrm{C}$ for $24 \mathrm{~h}$. After washing with PBS for three times, the plate was fixed with methanol. Excess methanol was sucked out; after drying at room temperature, we added $200 \mu \mathrm{L}$ $1 \%$ crystal violet solution and incubated at room temperature for $15 \mathrm{~min}$. The excess dye was sucked out and 
washed with PBS for 2-3 times. After drying at room temperature, $200 \mu \mathrm{l} 95 \%$ ethanol was added and incubated for $20 \mathrm{~min}$. The absorbance value was determined at $570 \mathrm{~nm}$.

\section{XTT dyeing}

XTT with a concentration of $0.2 \mathrm{mg} / \mathrm{mL}$ was prepared with PBS, and then the XTT solution was volumized with phenazine methyl ester to a concentration of $0.02 \mathrm{mg} /$ $\mathrm{mL}$. Next, $100 \mu \mathrm{L}$ of drug culture solution was added to each well of the 96-well plate; $100 \mu \mathrm{L}$ of overnight cultured bacteria solution was inoculated and incubated at $37^{\circ} \mathrm{C}$ for $24 \mathrm{~h}$. After washing the plate with PBS for three times, XTT solution was added and incubated at $37^{\circ} \mathrm{C}$ for $3 \mathrm{~h}$, and the absorbance value was determined at 490 nm.

\section{Mice model of MRSA pneumonia}

Twenty-five male Kunming mice at 8 weeks old were randomly divided into five groups: normal saline group, AgMOF group, vancomycin group, Ag-MOF-Vanc group and PLT@Ag-MOF-Vanc group. The mice were infected with $150 \mu \mathrm{L}$ MRSA (concentration $1 \times 10^{8} \mathrm{CFU} / \mathrm{mL}$ ) by tracheal injection. Eight hours after bacterial injection, the mice were given tail vein injection with $100 \mu \mathrm{L}$ corresponding drugs once a day for 3 consecutive days. HE staining of lung tissue was taken on the first, second, third, and fourth day of treatment to observe the alveolar structure and integrity of ciliated endothelial cells, inflammation, necrosis, and infiltration of inflammatory cells (macrophages) in the alveoli. On day 4, the lung tissues were stained by immunohistochemistry to detect the expression of inflammatory cytokines TNF- $\alpha$ and IL-6. The bacterial count of bronchoalveolar lavage fluid was detected. The whole blood of the mice was collected for blood test indicators (complete blood count, CRP). The levels of TNF- $\alpha$ and IL- 6 in blood were detected by ELISA. Another fifty mice with MRSA pneumonia were grouped and treated with corresponding drugs respectively. We recorded the survival status of mice and created the survival curve.

\section{In vivo imaging}

To evaluate the targeting ability of PLT@Ag-MOFVanc in vivo, mice model of MRSA pneumonia were injected with Cy5-labeled Ag-MOF-Vanc or Cy5labeled PLT@Ag-MOF-Vanc through the tail vein. The Xenogen IVIS Lumina XR imaging system (Caliper Life Sciences, USA) was used to evaluate fluorescence signals at 6, 24, and $48 \mathrm{~h}$ after administration. After $48 \mathrm{~h}$, the mice were killed by cervical dislocation; the brain, heart, liver, spleen, lung, and kidney were collected.
The fluorescence signal was further detected using the Xenogen IVIS Lumina XR imaging system (Caliper Life Sciences, USA).

\section{In vivo toxicity testing}

Healthy male Kunming mice at 8 weeks old were randomized into five groups $(\mathrm{n}=5)$ and injected with 100 $\mu \mathrm{L}$ of normal saline, Ag-MOF, vancomycin, Ag-MOFVanc, or PLT@Ag-MOF-Vanc via the tail vein. The mice body weight was measured everyday. One week later, we collected whole blood to test hematological and biochemical indicators (RBC, WBC, PLT, ALT, AST, BUN, CREA). The mice were killed through cervical dislocation. The major organs (heart, liver, spleen, lung, and kidney) were collected, stained with HE, and observed and photographed under microscope.

\section{Statistical analysis}

Data were assessed by GraphPad Prism software and expressed as mean $\pm \mathrm{SD}$. Intergroup differences were assessed by oneway ANOVA with subsequent Tukey's post-test. Significance was demonstrated by $\mathrm{p}<0.05$.

\section{Acknowledgements \\ Not applicable.}

\section{Authors' contributions}

$X M N, R G$, and $R H$ designed the study. $R H, G Q C, H T L, X S L, J D Z$, and JL performed the laboratory correlative experiments. JL analyzed data. $\mathrm{RH}$ wrote the manuscript. XLS and XSL edited the manuscript. All authors read and approved the final manuscript.

\section{Funding}

This project was supported by the National Natural Science Foundation of China $(81602801,81971748)$ and the Natural Science Foundation of Hunan Province (2019JJ40464).

\section{Availability of data and materials}

All data generated or analysed during this study are included in this published article.

\section{Declarations}

Ethics approval and consent to participate

Animal experiments were approved by the Experimental Animal Welfare Ethics Committee of Central South University (No. 2020sydw0109).

Consent for publication

Not applicable.

Conflict of interest

The authors declare that they have no competing interests.

\section{Author details}

${ }^{1}$ Department of Blood Transfusion, The Third Xiangya Hospital, Central South University, Hunan, Changsha, China. ${ }^{2}$ Department of Laboratory Medicine, The Third Xiangya Hospital, Central South University, Hunan, Changsha, China. ${ }^{3}$ Department of Orthopedics, Changsha Hospital of Traditional Chinese Medicine, Changsha Eighth Hospital, Hunan, Changsha, China.

Received: 24 April 2021 Accepted: 27 July 2021

Published online: 04 August 2021 


\section{References}

1. Thomer L, Schneewind O, Missiakas D. Pathogenesis of Staphylococcus aureus bloodstream infections. Annu Rev Pathol. 2016;11(1):343-64.

2. Bush K, Bradford PA. Epidemiology of $\beta$-lactamase-producing pathogens. Clin Microbiol Rev. 2020;33(2):e00047-19.

3. Peacock SJ, Paterson GK. Mechanisms of methicillin resistance in Staphylococcus aureus. Annu Rev Biochem. 2015;84:577-601.

4. Turner NA, Sharma-Kuinkel BK, Maskarinec SA, Eichenberger EM, Shah PP, Carugati M, Holland TL, Fowler VJ Jr. Methicillin-resistant Staphylococcus aureus: an overview of basic and clinical research. Nat Rev Microbiol. 2019;17(4):203-18.

5. Gauguet S, D'Ortona S, Ahnger-Pier K, Duan B, Surana NK, Lu R, CywesBentley C, Gadjeva M, Shan Q, Priebe GP, et al. Intestinal microbiota of mice influences resistance to Staphylococcus aureus pneumonia. Infect Immun. 2015;83(10):4003-14.

6. Lindsay JA. Hospital-associated MRSA and antibiotic resistance-what have we learned from genomics? Int J Med Microbiol. 2013;303(6-7):318-23.

7. Sarrou S, Malli E, Tsilipounidaki K, Florou Z, Medvecky M, Skoulakis A, Hrabak J, Papagiannitsis CC, Petinaki E. MLS-resistant in Central Greece: rate of resistance and molecular characterization. Microb Drug Resist. 2019;25(4):543-50.

8. Khosravi AD, Jenabi A, Montazeri EA. Distribution of genes encoding resistance to aminoglycoside modifying enzymes in methicillinresistant Staphylococcus aureus (MRSA) strains. Kaohsiung J Med Sci. 2017;33(12):587-93.

9. Fischer $\mathrm{AH}$, Haskin A, Okoye GA. Patterns of antimicrobial resistance in lesions of hidradenitis suppurativa. J Am Acad Dermatol. 2017;76(2):309-13.

10. Gajdács M. The continuing threat of methicillin-resistant. Antibiotics. 2019;8(2):52.

11. De Oliveira DMP, Forde BM, Kidd TJ, Harris PNA, Schembri MA, Beatson SA, Paterson DL, Walker MJ. Antimicrobial resistance in ESKAPE pathogens. Clin Microbiol Rev. 2020;33(3):e00181-19.

12. Ma YX, Wang CY, Li YY, Li J, Wan QQ, Chen JH, Tay FR, Niu LN. Considerations and Caveats in Combating ESKAPE Pathogens against Nosocomial Infections. Adv Sci. 2020;7(1):1901872.

13. Gerlach D, Guo Y, De Castro C, Kim SH, Schlatterer K, Xu FF, Pereira C, Seeberger PH, Ali S, Codée J, et al. Methicillin-resistant Staphylococcus aureus alters cell wall glycosylation to evade immunity. Nature. 2018;563(7733):705-9.

14. Watkins RR, Holubar M, David MZ. Antimicrobial resistance in methicillinresistant to newer antimicrobial agents. Antimicrob Agents Chemother. 2019;63(12):e01216-19.

15. Vestergaard $M$, Frees $D$, Ingmer $H$. Antibiotic resistance and the MRSA problem. Microbiol Spectr. 2019. https://doi.org/10.1128/microbiolspec. GPP3-0057-2018.

16. Álvarez R, López Cortés LE, Molina J, Cisneros JM, Pachón J. Optimizing the clinical use of vancomycin. Antimicrob Agents Chemother. 2016;60(5):2601-9.

17. Bruniera FR, Ferreira FM, Saviolli L, Bacci MR, Fonseca F. The use of vancomycin with its therapeutic and adverse effects: a review. Eur Rev Med Pharmacol Sci. 2015;19(4):694-700.

18. Gao Y, Chen Y, Cao Y, Mo A, Peng Q. Potentials of nanotechnology in treatment of methicillin-resistant Staphylococcus aureus. Eur J Med Chem. 2021;213:113056.

19. Gardete S, Tomasz A. Mechanisms of vancomycin resistance in Staphy/ococcus aureus. J Clin Invest. 2014;124(7):2836-40.

20. Canty E, Carnahan B, Curley T, Anususinha E, Hamdy RF, Ericson JE. Reduced vancomycin susceptibility, MRSA and treatment failure in pediatric Staphylococcus aureus Bloodstream Infections. Pediatr Infect Dis J. 2021;40(5):429-33.

21. Tong SYC, Lye DC, Yahav D, Sud A, Robinson JO, Nelson J, Archuleta S, Roberts MA, Cass A, Paterson DL, et al. Effect of vancomycin or daptomycin with vs without an antistaphylococcal $\beta$-lactam on mortality, bacteremia, relapse, or treatment failure in patients with MRSA bacteremia: a randomized clinical trial. JAMA. 2020;323(6):527-37.

22. Alosaimy S, Sabagha NL, Lagnf AM, Zasowski EJ, Morrisette T, Jorgensen SCJ, Trinh TD, Mynatt RP, Rybak MJ. Monotherapy with vancomycin or daptomycin versus combination therapy with $\beta$-lactams in the treatment of methicillin-resistant Staphylococcus Aureus bloodstream infections: a retrospective cohort analysis. Infect Dis Ther. 2020;9(2):325-39.
23. Fernández-González R, Díaz López MD, Lorenzo Vizcaya AM, González Noya A. Daptomycin associated eosinophilic pneumonia. Med Clin. 2021;156(3):148-9.

24. Liu C, Bayer A, Cosgrove SE, Daum RS, Fridkin SK, Gorwitz RJ, Kaplan SL, Karchmer AW, Levine DP, Murray BE, et al. Clinical practice guidelines by the infectious diseases society of america for the treatment of methicillinresistant Staphylococcus aureus infections in adults and children: executive summary. Clin Infect Dis. 2011;52(3):285-92.

25. Miller WR, Bayer AS, Arias CA. Mechanism of action and resistance to daptomycin in Staphylococcus aureus and Enterococci. Cold Spring Harb Perspect Med. 2016;6(11):a026997.

26. Yin Y, Chen H, Li S, Gao H, Sun S, Li H, Wang R, Jin L, Liu Y, Wang H. Daptomycin resistance in methicillin-resistant Staphylococcus aureus is conferred by IS256 insertion in the promoter of mprF along with mutations in mprF and walK. Int J Antimicrob Agents. 2019;54(6):673-80.

27. Kale-Pradhan PB, Giuliano C, Jongekrijg A, Rybak MJ. Combination of vancomycin or daptomycin and beta-lactam antibiotics: a meta-analysis. Pharmacotherapy. 2020;40(7):648-58.

28. Rashid M-U, Weintraub A, Nord CE. Effect of new antimicrobial agents on the ecological balance of human microflora. Anaerobe. 2012;18(2):249-53.

29. Vanamala K, Tatiparti K, Bhise K, Sau S, Scheetz MH, Rybak MJ, Andes D, lyer AK. Novel approaches for the treatment of methicillin-resistant Staphylococcus aureus: using nanoparticles to overcome multidrug resistance. Drug Discov Today. 2021;26(1):31-43.

30. Zhou K, Chao L, Chen D, Pan Y, Xie S. A review on nanosystems as an effective approach against infections of Staphylococcus aureus. Int J Nanomed. 2018;13:7333-47.

31. Sánchez-López E, Gomes D, Esteruelas G, Bonilla L, Lopez-Machado AL, Galindo R, Cano A, Espina M, Ettcheto M, Camins A, et al. Metal-based nanoparticles as antimicrobial agents: an overview. Nanomaterials. 2020;10(2):292.

32. Chernousova S, Epple M. Silver as antibacterial agent: ion, nanoparticle, and metal. Angew Chem Int Ed Engl. 2013;52(6):1636-53.

33. Bonilla-Gameros L, Chevallier P, Sarkissian A, Mantovani D. Silver-based antibacterial strategies for healthcare-associated infections: processes, challenges, and regulations. An integrated review. Nanomedicine. 2020;24:102142.

34. Yin IX, Zhang J, Zhao IS, Mei ML, Li Q, Chu CH. The antibacterial mechanism of silver nanoparticles and its application in dentistry. Int J Nanomed. 2020;15:2555-62.

35. Liao S, Zhang Y, Pan X, Zhu F, Jiang C, Liu Q, Cheng Z, Dai G, Wu G, Wang $L$, et al. Antibacterial activity and mechanism of silver nanoparticles against multidrug-resistant. Int J Nanomed. 2019;14:1469-87.

36. Mekkawy Al, El-Mokhtar MA, Nafady NA, Yousef N, Hamad MA, ElShanawany SM, Ibrahim EH, Elsabahy M. In vitro and in vivo evaluation of biologically synthesized silver nanoparticles for topical applications: effect of surface coating and loading into hydrogels. Int J Nanomed. 2017;12:759-77.

37. Kora AJ, Rastogi L. Enhancement of antibacterial activity of capped silver nanoparticles in combination with antibiotics, on model gram-negative and gram-positive bacteria. Bioinorg Chem Appl. 2013;2013:871097.

38. Chou TC. Theoretical basis, experimental design, and computerized simulation of synergism and antagonism in drug combination studies. Pharmacol Rev. 2006;58(3):621-81.

39. Hussain S, Joo J, Kang J, et al. Antibiotic-loaded nanoparticles targeted to the site of infection enhance antibacterial efficacy. Nat Biomed Eng. 2018;2(2):95-103.

40. Wieme L, Kresse SV. Tuning the balance between dispersion and entropy to design temperature-responsive flexible metal-organic frameworks. Nat Commun. 2018;9(1):4899.

41. Dehaini D, Wei X, Fang RH, Masson S, Zhang L. Erythrocyte-platelet hybrid membrane coating for enhanced nanoparticle functionalization. Adv Mater. 2017:29(16):201606209.

42. Hu CM, Fang RH, Wang KC, Luk BT, Thamphiwatana S, Dehaini D, Nguyen P, Angsantikul P, Wen CH, Kroll AV, et al. Nanoparticle biointerfacing by platelet membrane cloaking. Nature. 2015;526(7571):118-21.

43. Wei X, Gao J, Fang RH, Luk BT, Kroll AV, Dehaini D, Zhou J, Kim HW, Gao W, Lu W, et al. Nanoparticles camouflaged in platelet membrane coating as an antibody decoy for the treatment of immune thrombocytopenia. Biomaterials. 2016;111:116-23. 
44. Yeaman MR. Platelets in defense against bacterial pathogens. Cell Mol Life Sci. 2010;67(4):525-44.

45. Vieira-de-Abreu A, Campbell RA, Weyrich AS, Zimmerman GA. Platelets: versatile effector cells in hemostasis, inflammation, and the immune continuum. Semin Immunopathol. 2012;34(1):5-30

46. Mcfadyen JD, Kaplan ZS. Platelets are not just for clots. Transfus Med Rev. 2015:29(2):110-9.

47. Viela F, PrystopiukV, Leprince A, Mahillon J, Dufrêne Y. Binding of Staphylococcus aureus protein A to von Willebrand factor is regulated by mechanical force. mBio. 2019;10(2):e00555-19.

48. Fitzgerald JR, Foster TJ, Cox D. The interaction of bacterial pathogens with platelets. Nat Rev Microbiol. 2006;4(6):445-57.

49. Pei Y, Mohamed MF, Seleem MN, Yeo Y. Particle engineering for intracellular delivery of vancomycin to methicillin-resistant Staphylococcus aureus (MRSA)-infected macrophages. J Controll Release. 2017;267:133-43.

50. Su L, Zhang JH, Gomez H, Murugan R, Hong X, Xu D, Jiang F, Peng $Z Y$. Reactive oxygen species-induced lipid peroxidation in apoptosis, autophagy, and ferroptosis. Oxid Med Cell Longev. 2019;2019:5080843.

51. Cui Y, Zhao Y, Tian Y, Zhang W, Lü X, Jiang X. The molecular mechanism of action of bactericidal gold nanoparticles on Escherichia coli. Biomaterials. 2012;33(7):2327-33.
52. Zhao Y, Ye C, Liu W, Chen R, Jiang X. Tuning the composition of AuPt bimetallic nanoparticles for antibacterial application. Angew Chem Int Ed Engl. 2014;53(31):8127-31.

53. Marrache S, Dhar S. Engineering of blended nanoparticle platform for delivery of mitochondria-acting therapeutics. Proc Natl Acad Sci USA. 2012;109(40):16288-93.

54. Medina Cruz D, Mi G, Webster TJ. Synthesis and characterization of biogenic selenium nanoparticles with antimicrobial properties made by Staphylococcus aureus, methicillin-resistant Staphylococcus aureus (MRSA), Escherichia coli, and Pseudomonas aeruginosa. J Biomed Mater Res A. 2018;106(5):1400-12.

\section{Publisher's Note}

Springer Nature remains neutral with regard to jurisdictional claims in published maps and institutional affiliations.
Ready to submit your research? Choose BMC and benefit from:

- fast, convenient online submission

- thorough peer review by experienced researchers in your field

- rapid publication on acceptance

- support for research data, including large and complex data types

- gold Open Access which fosters wider collaboration and increased citations

- maximum visibility for your research: over $100 \mathrm{M}$ website views per year

At BMC, research is always in progress.

Learn more biomedcentral.com/submissions 